\title{
SELF-NORMALIZED LARGE DEVIATIONS
}

\author{
BY QI-MAN SHAO ${ }^{1}$ \\ University of Oregon
}

Let $\left\{X, X_{n}, n \geq 1\right\}$ be a sequence of independent and identically distributed random variables. The classical Cramér-Chernoff large deviation states that $\lim _{n \rightarrow \infty} n^{-1} \ln P\left(\left(\sum_{i=1}^{n} X_{i}\right) / n \geq x\right)=\ln \rho(x)$ if and only if the moment generating function of $X$ is finite in a right neighborhood of zero. This paper uses $n^{(p-1) / p} V_{n, p}=n^{(p-1) / p}\left(\sum_{i=1}^{n}\left|X_{i}\right|^{p}\right)^{1 / p}(p>1)$ as the normalizing constant to establish a self-normalized large deviation without any moment conditions. A self-normalized moderate deviation, that is, the asymptotic probability of $P\left(S_{n} / V_{n, p} \geq x_{n}\right)$ for $x_{n}=o\left(n^{(p-1) / p}\right)$, is also found for any $X$ in the domain of attraction of a normal or stable law. As a consequence, a precise constant in the self-normalized law of the iterated logarithm of Griffin and Kuelbs is obtained. Applications to the limit distribution of self-normalized sums, the asymptotic probability of the $t$ statistic as well as to the Erdős-Rényi-Shepp law of large numbers are also discussed.

1. Introduction. Throughout this paper, let $(\Omega, \Sigma, P)$ denote a probability space, and let $\left\{X, X_{n}, n \geq 1\right\}$ be a sequence of independent and identically distributed (i.i.d.) nondegenerate real-valued random variables on the probability space. Put

$$
S_{n}=\sum_{i=1}^{n} X_{i}, \quad V_{n}^{2}=\sum_{i=1}^{n} X_{i}^{2}, \quad n=1,2, \ldots
$$

The classical Cramér-Chernoff large deviation [Chernoff (1952)] states that if (A)

$$
E e^{t_{0} X}<\infty \text { for some } t_{0}>0,
$$

then for every $x>E X$,

$$
\lim _{n \rightarrow \infty} n^{-1} \ln P\left(\frac{S_{n}}{n} \geq x\right)=\ln \rho(x)
$$

or equivalently,

$$
\lim _{n \rightarrow \infty} P\left(\frac{S_{n}}{n} \geq x\right)^{1 / n}=\rho(x)
$$

where $\rho(x)=\inf _{t \geq 0} e^{-t x} E e^{t X}$.

Roughly speaking, this type of large deviation shows that the convergence rate in the law of large numbers is exponential if the moment generating

Received April 1995; revised J une 1996.

${ }^{1}$ Research partially supported by a National University of Singapore Research Project.

AMS 1991 subject classifications. Primary 60F 10, 60F 15; secondary 60G50, 62E 20.

Key words and phrases. Self-normalized partial sums, large deviation, moderate deviation, law of the iterated logarithm, the Erdös-Rényi-Shepp law of large numbers, limit distribution, $t$-statistic. 
function is finite in a right neighborhood of zero. The latter is also necessary for an exponential scale [Petrov and Širokova (1973)]. Essentially built on condition (A), the area of large deviations in finite-dimensional spaces and even in abstract spaces has been well developed, and various applications in statistics [cf. Bahadur (1971)], engineering, statistical mechanics and applied probability have been found in recent years. We refer to de Acosta (1988), Stroock (1984), Donsker and Varadhan (1987) and Dembo and Zeitouni (1992) and references therein for more details.

On the other hand, the so-called self-normalized limit theorems put a totally new countenance upon classical limit theorems. I n contrast to the well-known Hartman-Wintner law of the iterated logarithm (LIL) and its converse by Strassen (1966), Griffin and Kuelbs (1989) obtained a self-normalized law of the iterated logarithm for all distributions in the domain of attraction of a normal or stable law.

1. If $E X=0$ and $E X^{2} I\{|X| \leq x\}$ is slowly varying as $x \rightarrow \infty$, then

$$
\limsup _{n \rightarrow \infty} \frac{S_{n}}{V_{n}(2 \log \log n)^{1 / 2}}=1 \text { a.s. }
$$

2. If $X$ is symmetric and in the domain of attraction of a stablelaw, then there is a positive constant $C$ such that

$$
\limsup _{n \rightarrow \infty} \frac{S_{n}}{V_{n}(2 \log \log n)^{1 / 2}}=C \text { a.s. }
$$

It should be noted that under (2),

$$
\limsup _{n \rightarrow \infty} \frac{S_{n}}{a_{n}}=0 \text { or } \infty \text { a.s. }
$$

for any sequence $\left\{a_{n}, n \geq 1\right\}$ of positive numbers with $a_{n} \rightarrow \infty$ (Lévy and Marcinkiewicz [see Chung (1974), page 131]). So, the significance of the above result is obvious. It shows that when the normalizing constants in the classical limit theorem are replaced by an appropriate sequence of random variables, a similar result to the classical limit theorem may still hold under less or even without any moment conditions. This naturally leads to the exploration of the feasibility of a self-normalized large deviation, which should be interesting within the probability theory itself as well as for applications to other fields. The main aim of this paper is to establish such a self-normalized large deviation for arbitrary random variables without any moment conditions.

Theorem 1.1. Assume that either $E X \geq 0$ or $E X^{2}=\infty$. Then

$$
\lim _{n \rightarrow \infty} P\left(\frac{S_{n}}{V_{n} n^{1 / 2}} \geq x\right)^{1 / n}=\operatorname{supinf}_{c \geq 0} E \exp \left(t\left(c X-x\left(X^{2}+c^{2}\right) / 2\right)\right)
$$

for $x>E X /\left(E X^{2}\right)^{1 / 2}$, where $E X /\left(E X^{2}\right)^{1 / 2}$ is interpreted to bezero if $E X^{2}=\infty$, and $0 / 0$ to be $\infty$. 
More generally, using $\left(\sum_{i=1}^{n}\left|X_{i}\right|^{p}\right)^{1 / p} n^{1-1 / p}, p>1$ as normalizing constants, we have the following.

Theorem 1.2. Let $p>1$. Assume that either $E X \geq 0$ or $E|X|^{p}=\infty$. Then

$$
\begin{aligned}
\lim _{n \rightarrow \infty} & P\left(\frac{S_{n}}{V_{n, p} n^{1-1 / p}} \geq x\right)^{1 / n} \\
\quad & \operatorname{supinf}_{c \geq 0} E \exp \left(t\left(c X-x\left(\frac{1}{p}|X|^{p}+\frac{p-1}{p} c^{p /(p-1)}\right)\right)\right)
\end{aligned}
$$

for $x>E X /\left(E|X|^{p}\right)^{1 / p}$, where $V_{n, p}=\left(\sum_{i=1}^{n}\left|X_{i}\right|^{p}\right)^{1 / p}$ and $E X /\left(E|X|^{p}\right)^{1 / p}=0$ if $E|X|^{p}=\infty$.

From Theorem 1.1 the corollary follows immediately.

Corollary 1.1. Assume that either $E X=0$ or $E X^{2}=\infty$. Then

$$
\lim _{n \rightarrow \infty} P\left(\frac{S_{n}}{V_{n} n^{1 / 2}} \geq x\right)^{1 / n}=\operatorname{supinf}_{c \geq 0} E \exp \left(t\left(\frac{c X-x\left(X^{2}+c^{2}\right)}{2}\right)\right)
$$

for $x>0$.

REMARK 1.1. Note that for any random variable $X$ either $E X^{2}<\infty$ or $E X^{2}=\infty$. If $E X^{2}<\infty$, which obviously implies $E|X|<\infty$, the assumption that $E X \geq 0$ in Theorem 1.1 is reasonable. In other words, Theorem 1.1 holds without assuming any moment conditions.

REMARK 1.2. If $E X^{2}<\infty$ and $E X<0$, one can see from the proof of Theorem 1.1 that (1.3) remains valid for $x>0$.

REMARK 1.3. From the Cauchy inequality, it follows that

$$
S_{n} /\left(V_{n} n^{1 / 2}\right) \leq 1 \text { if } V_{n}>0
$$

and it is easy to see that both sides of (1.3), (1.4) and (1.5) are equal to $P(X=0)$ for $x>1$.

We will give proofs of these results in the next section. Based on similar ideas, Section 3 presents self-normalized moderate deviations (Theorems 3.13.3), which, in turn, enable us to get the exact constant $C$ in (1.2) (Theorem 5.1). As another application of Theorem 3.2, Section 6 settles a conjecture of Logan, Mallows, Rice and Shepp (1973). Application to the $t$-statistic is discussed in Section 7. As a direct application of Theorem 1.1, Section 8 deals with a self-normalized Erdős-Rényi-Shepp type law of large numbers without any moment conditions (Theorem 8.1). 


\section{Proofs of Theorems 1.1 and 1.2 .}

Proof OF ThEOREM 1.1. The main idea of the proof is to reduce the problem to that of Cramér-Chernoff large deviation, by using the following wellknown fact: for any positive numbers $x$ and $y$,

$$
x y=\inf _{b>0} \frac{1}{2}\left(\frac{x^{2}}{b}+y^{2} b\right) \text {. }
$$

By (2.1), we have

$$
V_{n} n^{1 / 2}=\inf _{b>0} \frac{1}{2 b}\left(V_{n}^{2}+n b^{2}\right) \text { if } V_{n}>0
$$

and

$$
\begin{aligned}
P\left(\frac{S_{n}}{V_{n} n^{1 / 2}} \geq x\right) & =P\left(S_{n} \geq x \inf _{b>0} \frac{1}{2 b}\left(V_{n}^{2}+n b^{2}\right) \text { or } V_{n}=0\right) \\
& =P\left(\sup _{b>0} \sum_{i=1}^{n}\left(b X_{i}-x\left(X_{i}^{2}+b^{2}\right) / 2\right) \geq 0 \text { or } V_{n}=0\right) \\
& =P\left(\sup _{b \geq 0} \sum_{i=1}^{n}\left(b X_{i}-x\left(X_{i}^{2}+b^{2}\right) / 2\right) \geq 0\right)
\end{aligned}
$$

Note that for $x>E X /\left(E X^{2}\right)^{1 / 2}(\geq 0)$ and for $b \geq 0$,

$$
E \exp \left(t\left(b X-x\left(X^{2}+b^{2}\right) / 2\right)\right)<\infty \text { for all } t \geq 0
$$

and

$$
\begin{aligned}
E\left(b X-x\left(X^{2}+b^{2}\right) / 2\right) & \text { if } E X^{2}=\infty, \\
& = \begin{cases}-\infty, & \text { if } E X^{2}<\infty . \\
-(x / 2)(b-(E X) / x)^{2}-\frac{1}{2}\left(x E X^{2}-(E X)^{2} / x\right)<0, & \end{cases}
\end{aligned}
$$

Thus, by (2.3) and (1.1),

$$
\begin{aligned}
& \liminf _{n \rightarrow \infty} P\left(\frac{S_{n}}{V_{n} n^{1 / 2}} \geq x\right)^{1 / n} \\
& \quad \geq \liminf _{n \rightarrow \infty} \sup _{b \geq 0} P\left(\sum_{i=1}^{n}\left(b X_{i}-x\left(X_{i}^{2}+b^{2}\right) / 2\right) \geq 0\right)^{1 / n} \\
& \quad \geq \operatorname{supinf}_{b \geq 0} E \exp \left(t\left(b X-\frac{x\left(X^{2}+b^{2}\right)}{2}\right)\right) .
\end{aligned}
$$

To finish the proof of (1.3), it suffices to show that

(2.5) $\quad \limsup _{n \rightarrow \infty} P\left(\frac{S_{n}}{V_{n} n^{1 / 2}} \geq x\right)^{1 / n} \leq \operatorname{supinf}_{b \geq 0} E \exp \left(t\left(b X-\frac{x\left(X^{2}+b^{2}\right)}{2}\right)\right)$. 
Recalling (2.3), for $A>2$

$$
\begin{aligned}
P\left(\frac{S_{n}}{V_{n} n^{1 / 2}} \geq x\right) \leq & P\left(\sup _{b>4 A} \sum_{i=1}^{n}\left(b X_{i}-\frac{x\left(X_{i}^{2}+b^{2}\right)}{2}\right) \geq 0\right) \\
& +P\left(\sup _{0 \leq b \leq 4 A} \sum_{i=1}^{n}\left(b X_{i}-\frac{x\left(X_{i}^{2}+b^{2}\right)}{2}\right) \geq 0\right) \\
:= & I_{1}+I_{2} .
\end{aligned}
$$

Notice that

$$
\begin{aligned}
I_{1} & =P\left(\operatorname { s u p } _ { b > 4 A } \sum _ { i = 1 } ^ { n } \left(b X_{i} I\left\{\left|X_{i}\right| \leq x A\right\}+b X_{i} I\left\{\left|X_{i}\right|>x A\right\}\right.\right. \\
& \left.\left.-\frac{x\left(X_{i}^{2}+b^{2}\right)}{2}\right) \geq 0\right) \\
& \leq P\left(\sup _{b>4 A} \sum_{i=1}^{n}\left(b x A+b X_{i} I\left\{\left|X_{i}\right|>x A\right\}-\frac{x\left(X_{i}^{2}+b^{2}\right)}{2}\right) \geq 0\right) \\
& \leq P\left(\sup _{b>4 A} \sum_{i=1}^{n}\left(b X_{i} I\left\{\left|X_{i}\right|>x A\right\}-\frac{x\left(X_{i}^{2}+b^{2} / 2\right)}{2}\right) \geq 0\right) \\
& =P\left(\sum_{i=1}^{n} X_{i} I\left\{\left|X_{i}\right|>x A\right\} \geq \frac{x}{2} \inf _{b>4 A}\left(\frac{\sum_{i=1}^{n} X_{i}^{2}}{b}+\frac{b n}{2}\right)\right) \\
& \leq P\left(\sum_{i=1}^{n} X_{i} I\left\{\left|X_{i}\right|>x A\right\} \geq \frac{x}{\sqrt{2}}\left(n \sum_{i=1}^{n} X_{i}^{2}\right)^{1 / 2}, V_{n}>0\right) \\
& \leq P\left(\sum_{i=1}^{n} I\left\{\left|X_{i}\right|>x A\right\} \geq \frac{x^{2}}{2} n\right)
\end{aligned}
$$

by the Cauchy inequality. Applying the Chernoff large deviation to the binomial random variable $B(n, p)$, it follows that for all $a>0$,

$$
P(B(n, p)>a n) \leq\left(\frac{e p}{a}\right)^{a n} .
$$

Therefore

$$
P\left(\sum_{i=1}^{n} I\left\{\left|X_{i}\right|>x A\right\} \geq \frac{x^{2}}{2} n\right) \leq\left(\frac{6 P(|X|>x A)}{x^{2}}\right)^{x^{2} n / 2},
$$

which together with (2.7) yields

$$
\limsup _{n \rightarrow \infty} I_{1}^{1 / n} \leq\left(\frac{6 P(|X|>x A)}{x^{2}}\right)^{x^{2} / 2} .
$$

We next estimate $I_{2}$. Take $A \geq 2$ such that

$$
P(|X| \leq A)>1 / 2 \text {. }
$$


Let

$$
0<\delta<1, \quad \Delta:=\Delta(A, \delta)=\frac{\delta^{2}}{(10+60 x) A^{4}}
$$

and let $Y$ be a standard normal random variable independent of $X$. We have

$$
\begin{aligned}
I_{2} & \leq P\left(\max _{1 \leq j \leq 1+4 A / \Delta} \sup _{(j-1) \Delta \leq b \leq j \Delta} \sum_{i=1}^{n}\left(b X_{i}-x\left(X_{i}^{2}+b^{2}\right) / 2\right) \geq 0\right) \\
& \leq P\left(\max _{1 \leq j \leq 1+4 A / \Delta} \sum_{i=1}^{n}\left(j \Delta X_{i}-x\left(X_{i}^{2}+((j-1) \Delta)^{2}\right) / 2\right) \geq 0\right) \\
& \leq \sum_{1 \leq j \leq 1+4 A / \Delta} P\left(\sum_{i=1}^{n}\left(j \Delta X_{i}-x\left(X_{i}^{2}+((j-1) \Delta)^{2}\right) / 2\right) \geq 0\right) \\
& \leq \sum_{1 \leq j \leq 1+4 A / \Delta}\left(\inf _{t \geq 0} E \exp \left(t\left(j \Delta X-x\left(X^{2}+((j-1) \Delta)^{2}\right) / 2\right)\right)\right)^{n} \\
& \leq \sum_{1 \leq j \leq 1+4 A / \Delta}\left(\inf _{t \geq 0} \exp \left(t^{2} \delta^{2} / 2\right) E \exp \left(t\left(j \Delta X-x\left(X^{2}+((j-1) \Delta)^{2}\right) / 2\right)\right)\right)^{n} \\
& =\sum_{1 \leq j \leq 1+4 A / \Delta}\left(\inf _{t \geq 0} E \exp \left(t\left(j \Delta X+\delta Y-x\left(X^{2}+((j-1) \Delta)^{2}\right) / 2\right)\right)\right)^{n} .
\end{aligned}
$$

Put

$$
\xi_{j}=j \Delta X+\delta Y-x\left(X^{2}+(j \Delta)^{2}\right) / 2, \quad 1 \leq j \leq 1+4 A / \Delta .
$$

It is easy to see that $P\left(\xi_{j}=y\right)=0$ and $0<P\left(\xi_{j}<y\right)<1$ for any $y$ and that $-\infty \leq E \xi_{j}<0$. Therefore, in terms of Lemmas 1 and 3 of Chernoff (1952), there is $0<t_{j}<\infty$ such that

$$
E \exp \left(t_{j} \xi_{j}\right)=\inf _{t \geq 0} E \exp \left(t \xi_{j}\right) \leq 1 .
$$

As to $t_{j}$, by (2.12) and (2.10),

$$
\begin{aligned}
1 & \geq E \exp \left(t_{j}\left(j \Delta X+\delta Y-x\left(X^{2}+(j \Delta)^{2}\right) / 2\right)\right) \\
& \geq E \exp \left(t_{j}\left(j \Delta X+\delta Y-x\left(X^{2}+(j \Delta)^{2}\right) / 2\right)\right) I\{|X| \leq A\} \\
& =\exp \left(\left(\delta t_{j}\right)^{2} / 2\right) E \exp \left(t_{j}\left(j \Delta X-x\left(X^{2}+(j \Delta)^{2}\right) / 2\right)\right) I\{|X| \leq A\} \\
& \geq \exp \left(\left(\delta t_{j}\right)^{2} / 2\right) \exp \left(-t_{j}\left(j \Delta A+x\left(A^{2}+(j \Delta)^{2}\right) / 2\right)-1\right) \\
& \geq \exp \left(\left(\delta t_{j}\right)^{2} / 2\right) \exp \left(-t_{j}(4.5+30 x) A^{2}-1\right) \\
& \geq \exp \left(\frac{1}{2}\left\{\left(\delta t_{j}-(4.5+30 x) A^{2} / \delta\right)^{2}-\left((5.5+30 x) A^{2} / \delta\right)^{2}\right\}\right)
\end{aligned}
$$

for $1 \leq j \leq 1+4 A / \Delta$, which yields immediately

$$
t_{j} \leq(10+60 x) A^{2} / \delta^{2} \text { for } 1 \leq j \leq 1+4 A / \Delta .
$$


Therefore, by (2.11), (2.12) and (2.13),

$$
\begin{aligned}
& I_{2} \leq \sum_{1 \leq j \leq 1+4 A / \Delta}\left(E \exp \left(t_{j}\left(j \Delta X+\delta Y-x\left(X^{2}+((j-1) \Delta)^{2}\right) / 2\right)\right)\right)^{n} \\
& =\sum_{1 \leq j \leq 1+4 A / \Delta}\left(\exp \left(t_{j} x\left(j^{2}-(j-1)^{2}\right) \Delta^{2} / 2\right) E \exp \left(t_{j} \xi_{j}\right)\right)^{n} \\
& \leq \sum_{1 \leq j \leq 1+4 A / \Delta}\left(\exp \left(t_{j} j \Delta^{2}\right) \inf _{t \geq 0} E \exp \left(t \xi_{j}\right)\right)^{n} \\
& \leq(1+4 A / \Delta)\left(\exp \left(\Delta(1+4 A)(10+60 x) A^{2} / \delta^{2}\right)\right. \\
& \left.\quad \quad \quad \quad \sup _{b \geq 0} \inf _{t \geq 0} E \exp \left(t\left(b X+\delta Y-x\left(X^{2}+b^{2}\right) / 2\right)\right)\right)^{n} \\
& \leq(1+4 A / \Delta)\left(\exp (5 / A) \operatorname{supinf}_{b \geq 0} E \exp \left(t\left(b X+\delta Y-x\left(X^{2}+b^{2}\right) / 2\right)\right)\right)^{n} .
\end{aligned}
$$

It follows from (2.6), (2.9) and (2.14) that

$$
\begin{aligned}
\limsup _{n \rightarrow \infty} & P\left(\frac{S_{n}}{V_{n} n^{1 / 2}} \geq x\right)^{1 / n} \\
\leq & \left(\frac{6 P(|X|>x A)}{x^{2}}\right)^{x^{2} / 4} \\
& +\exp (5 / A) \operatorname{supinf}_{b \geq 0} E \exp \left(t\left(b X+\delta Y-\frac{x\left(X^{2}+b^{2}\right)}{2}\right)\right)
\end{aligned}
$$

for any $0<\delta<1$ and for any $A$ satisfying (2.10). Letting $A \rightarrow \infty$ leads to

$$
\begin{array}{rl}
\underset{n \rightarrow \infty}{\limsup } P & P\left(\frac{S_{n}}{V_{n} n^{1 / 2}} \geq x\right)^{1 / n} \\
\quad \leq \operatorname{supinf}_{b \geq 0} \exp \left((t \delta)^{2} / 2\right) E \exp \left(t\left(b X-\frac{x\left(X^{2}+b^{2}\right)}{2}\right)\right)
\end{array}
$$

for any $0<\delta<1$.

Clearly, (2.5) will be an immediate consequence of (2.15) and the following Lemma 2.1. This completes the proof of Theorem 1.1.

LEMMA 2.1. For any random variable $X$ we have

$$
\begin{aligned}
& \operatorname{limsupinf}_{\delta \downarrow 0} \exp \left((t \delta)^{2} / 2\right) E \exp \left(t\left(b X-x\left(X^{2}+b^{2}\right) / 2\right)\right) \\
& =\sup _{b \geq 0} \inf _{t \geq 0} E \exp \left(t\left(b X-x\left(X^{2}+b^{2}\right) / 2\right)\right)
\end{aligned}
$$

for $x>E X /\left(E X^{2}\right)^{1 / 2}$. Moreover, the convergence is uniform in $x \in[a, 1]$ for any $E X /\left(E X^{2}\right)^{1 / 2}<a<1$. 
The proof is given in the Appendix.

From the above proof of Theorem 1.1, one can obtain the following more general result.

THEOREM 2.1. Let $\mu$ and $\nu$ betwo real numbers. Assumethat either $E X \geq \mu$ or $E X^{2}=\infty$. Then

$$
\begin{aligned}
\lim _{n \rightarrow \infty} & P\left(\frac{\sum_{i=1}^{n}\left(X_{i}-\mu\right)}{\left(n \sum_{i=1}^{n}\left(X_{i}-\nu\right)^{2}\right)^{1 / 2}} \geq x\right)^{1 / n} \\
\quad= & \operatorname{supinf}_{c \geq 0} E \exp \left(t\left(c(X-\mu)-\frac{x\left((X-\nu)^{2}+c^{2}\right)}{2}\right)\right)
\end{aligned}
$$

for $x>(E X-\mu) / \sqrt{E(X-\nu)^{2}}$.

Proof of Theorem 1.2. Let $p>1$. It is well known that

$$
x^{1 / p} y^{1-1 / p}=\inf _{b>0}\left(\frac{1}{p} \frac{x}{b}+\frac{p-1}{p} y b^{1 /(p-1)}\right) \text { for any } x>0, y>0 .
$$

The remaining part of the proof is along the same lines as that of Theorem 1.1 , just by using (2.18) instead of (2.1), so the details are omitted here.

3. Self-normalized moderate deviations. Let $\left\{x_{n}, n \geq 1\right\}$ be a sequence of positive numbers with $x_{n} \rightarrow \infty$ as $n \rightarrow \infty$. Essentially, Theorem 1.1 gives us the asymptotic probability of $P\left(S_{n} \geq x_{n} V_{n}\right)$ when $x_{n} \asymp \sqrt{n}$. A natural question is whether we have an analogous result for general $\left\{x_{n}, n \geq 1\right\}$ without any moment conditions. The following theorems give an affirmative answer to this question.

THEOREM 3.1. Let $\left\{x_{n}, n \geq 1\right\}$ bea sequence of positivenumbers with $x_{n} \rightarrow$ $\infty$ and $x_{n}=o(\sqrt{n})$ as $n \rightarrow \infty$. If $E X=0$ and $E X^{2} I\{|X| \leq x\}$ is sl owly varying as $x \rightarrow \infty$, then

$$
\lim _{n \rightarrow \infty} x_{n}^{-2} \ln P\left(\frac{S_{n}}{V_{n}} \geq x_{n}\right)=-\frac{1}{2}
$$

The result is closely related to the Cramér (1938) large deviation. It is known [cf. Petrov (1975)] that

$$
\lim _{n \rightarrow \infty} x_{n}^{-2} \ln P\left(\frac{\left|S_{n}\right|}{\sqrt{n}} \geq x_{n}\right)=-\frac{1}{2}
$$

holds for any sequence of $\left\{x_{n}\right\}$ with $x_{n} \rightarrow \infty$ and $x_{n}=o(\sqrt{n})$ if and only if $E X=0, E X^{2}=1$ and $E \exp \left(t_{0}|X|\right)<\infty$ for some $t_{0}>0$. Theorem 3.1 shows 
again that the situation is quite different in the self-normalized limit theorems. It tells us that the main term of the asymptotic probability of $P\left(S_{n} \geq\right.$ $\left.x_{n} V_{n}\right)$ is distribution free as long as $X$ is in the domain of attraction of a normal law and $x_{n}=o(\sqrt{n})$. Our next theorem demonstrates that $P\left(S_{n} \geq x_{n} V_{n}\right)$ has the same exponent power up to a constant when $X$ is in the domain of attraction of a stable law.

THEOREM 3.2. Let $\left\{x_{n}, n \geq 1\right\}$ be a sequence of positive numbers with $x_{n} \rightarrow \infty$ and $x_{n}=o(\sqrt{n})$ as $n \rightarrow \infty$. Assume that there exist $0<\alpha<2, c_{1} \geq$ $0, c_{2} \geq 0, c_{1}+c_{2}>0$ and a slowly varying function $h(x)$ such that

$$
\begin{gathered}
P(X \geq x)=\frac{c_{1}+o(1)}{x^{\alpha}} h(x) \quad \text { and } \\
P(X \leq-x)=\frac{c_{2}+o(1)}{x^{\alpha}} h(x) \quad \text { as } x \rightarrow \infty .
\end{gathered}
$$

Moreover, assume that $E X=0$ if $1<\alpha<2, X$ is symmetric if $\alpha=1$ and that $c_{1}>0$ if $0<\alpha<1$. Then, we have

$$
\lim _{n \rightarrow \infty} x_{n}^{-2} \ln P\left(\frac{S_{n}}{V_{n}} \geq x_{n}\right)=-\beta\left(\alpha, c_{1}, c_{2}\right),
$$

where $\beta\left(\alpha, c_{1}, c_{2}\right)$ is the solution of $\Gamma\left(\beta, \alpha, c_{1}, c_{2}\right)=0$ and

$$
\begin{aligned}
& \Gamma\left(\beta, \alpha, c_{1}, c_{2}\right) \\
& \quad \begin{cases}c_{1} \int_{0}^{\infty} \frac{1+2 x-\exp \left(2 x-x^{2} / \beta\right)}{x^{\alpha+1}} d x & \text { if } 1<\alpha<2, \\
+c_{2} \int_{0}^{\infty} \frac{1-2 x-\exp \left(-2 x-x^{2} / \beta\right)}{x^{\alpha+1}} d x, & \text { if } \alpha=1, \\
c_{1} \int_{0}^{\infty} \frac{2-\exp \left(2 x-x^{2} / \beta\right)-\exp \left(-2 x-x^{2} / \beta\right)}{x^{2}} d x, & \\
c_{1} \int_{0}^{\infty} \frac{1-\exp \left(2 x-x^{2} / \beta\right)}{x^{\alpha+1}} d x & \text { if } 0<\alpha<1 . \\
+c_{2} \int_{0}^{\infty} \frac{1-\exp \left(-2 x-x^{2} / \beta\right)}{x^{\alpha+1}} d x, & \end{cases}
\end{aligned}
$$

In particular, if $X$ is symmetric, then

$$
\lim _{n \rightarrow \infty} x_{n}^{-2} \ln P\left(\frac{S_{n}}{V_{n}} \geq x_{n}\right)=-\beta(\alpha),
$$

where $\beta(\alpha)$ is the solution of

$$
\int_{0}^{\infty} \frac{2-\exp \left(2 x-x^{2} / \beta\right)-\exp \left(-2 x-x^{2} / \beta\right)}{x^{\alpha+1}} d x=0 .
$$


More generally, corresponding to Theorem 1.2, we have Theorem 3.3.

TheOREM 3.3. Assumethat there exist $0<\alpha<2, c_{1} \geq 0, c_{2} \geq 0, c_{1}+c_{2}>0$ and a slowly varying function $h(x)$ such that (3.2) holds. Moreover, assume that $E X=0$ if $1<\alpha<2, X$ is symmetric if $\alpha=1$ and that $c_{1}>0$ if $0<\alpha<1$. Let $p>\max (1, \alpha)$, and let $\left\{x_{n}, n \geq 1\right\}$ be a sequence of positive numbers with $x_{n} \rightarrow \infty$ and $x_{n}=o\left(n^{(p-1) / p}\right)$ as $n \rightarrow \infty$. Then, we have

$$
\lim _{n \rightarrow \infty} x_{n}^{-p /(p-1)} \ln P\left(\frac{S_{n}}{V_{n, p}} \geq x_{n}\right)=-(p-1) \beta_{p}\left(\alpha, c_{1}, c_{2}\right),
$$

where $\beta_{p}\left(\alpha, c_{1}, c_{2}\right)$ is the solution of $\Gamma_{p}\left(\beta, \alpha, c_{1}, c_{2}\right)=0$ and

$$
\begin{aligned}
& \Gamma_{p}\left(\beta, \alpha, c_{1}, c_{2}\right) \\
& \text { (3.7) }= \begin{cases}c_{1} \int_{0}^{\infty} \frac{1+p x-\exp \left(p x-x^{p} / \beta^{p-1}\right)}{x^{\alpha+1}} d x & \text { if } 1<\alpha<2, \\
+c_{2} \int_{0}^{\infty} \frac{1-p x-\exp \left(-p x-x^{p} / \beta^{p-1}\right)}{x^{\alpha+1}} d x, & \text { if } \alpha=1, \\
c_{1} \int_{0}^{\infty} \frac{2-\exp \left(p x-x^{p} / \beta^{p-1}\right)-\exp \left(-p x-x^{p} / \beta^{p-1}\right)}{x^{2}} d x, \\
c_{1} \int_{0}^{\infty} \frac{1-\exp \left(p x-x^{p} / \beta^{p-1}\right)}{x^{\alpha+1}} d x & \text { if } 0<\alpha<1 . \\
+c_{2} \int_{0}^{\infty} \frac{1-\exp \left(-p x-x^{p} / \beta^{p-1}\right)}{x^{\alpha+1}} d x, & \end{cases}
\end{aligned}
$$

In particular, if $X$ is symmetric, then

$$
\lim _{n \rightarrow \infty} x_{n}^{-p /(p-1)} \ln P\left(\frac{S_{n}}{V_{n, p}} \geq x_{n}\right)=-(p-1) \beta_{p}(\alpha),
$$

where $\beta_{p}(\alpha)$ is the solution of

$$
\int_{0}^{\infty} \frac{2-\exp \left(p x-x^{p} / \beta^{p-1}\right)-\exp \left(-p x-x^{p} / \beta^{p-1}\right)}{x^{\alpha+1}} d x=0 .
$$

REMARK 3.1. It is easy to see that $\Gamma_{p}\left(\beta, \alpha, c_{1}, c_{2}\right)$ is strictly decreasing and continuous on $(0, \infty)$ and by the l'Hôpital rule that

$$
\lim _{\beta \downarrow 0} \Gamma_{p}\left(\beta, \alpha, c_{1}, c_{2}\right)=\infty \text { and } \lim _{\beta \uparrow \infty} \Gamma_{p}\left(\beta, \alpha, c_{1}, c_{2}\right)=-\infty
$$

So, the solution of $\Gamma_{p}\left(\beta, \alpha, c_{1}, c_{2}\right)=0$ exists and is unique. 
4. Proofs of Theorems 3.1, 3.2 and 3.3. Recalling that a positive function $h(x)$ defined on $x \geq a$ for some $a \geq 0$ is said to be slowly varying (at $\infty$ ) if for all $t>0$,

$$
\lim _{x \rightarrow \infty} \frac{h(t x)}{h(x)}=1
$$

The following properties of a slowly varying function $h(x)$ are well known [cf. Karamata (1933), Feller (1966) and Bingham, Goldie and Teugels (1987)] and will be utilized in the following proofs.

(H1) $h(x)$ is representable in the form $h(x)=c(x) \exp \left(\int_{1}^{x}(a(y) / y) d y\right)$, where $c(x) \rightarrow c>0$, for some $c$, and $a(x) \rightarrow 0$ as $x \rightarrow \infty$.

(H2) For $0<c<C<\infty, \lim _{x \rightarrow \infty}(h(t x)) / h(x)=1$ uniformly in $c \leq t \leq C$.

(H3) $\forall \varepsilon>0, \lim _{x \rightarrow \infty} x^{-\varepsilon} h(x)=0$ and $\lim _{x \rightarrow \infty} x^{\varepsilon} h(x)=\infty$.

(H4) For any $\varepsilon>0$, there exists $x_{0}$ such that for all $x, x t \geq x_{0}$,

$$
\begin{gathered}
(1-\varepsilon)\left(t \vee \frac{1}{t}\right)^{-\varepsilon} \leq \frac{h(t x)}{h(x)} \leq(1+\varepsilon)\left(t \vee \frac{1}{t}\right)^{\varepsilon}, \\
\left|\frac{h(t x)}{h(x)}-1\right| \leq 2\left(\left(t \vee \frac{1}{t}\right)^{\varepsilon}-1\right) .
\end{gathered}
$$

(H5) For any $\theta>-1, \int_{a}^{x} y^{\theta} h(y) d y \sim\left(x^{\theta+1} h(x)\right) /(\theta+1)$ as $x \rightarrow \infty$.

Proof of Theorem 3.1. It suffices to show that

$$
\limsup _{n \rightarrow \infty} x_{n}^{-2} \ln P\left(\frac{S_{n}}{V_{n}} \geq x_{n}\right) \leq-\frac{1}{2}
$$

and

$$
\liminf _{n \rightarrow \infty} x_{n}^{-2} \ln P\left(\frac{S_{n}}{V_{n}} \geq x_{n}\right) \geq-\frac{1}{2} .
$$

The idea of the proof of (4.1) comes from Griffin and Kuelbs (1989). Put

$$
\begin{aligned}
l(x) & =E X^{2} I\{|X| \leq x\}, \quad b=\inf \{x \geq 1: l(x)>0\}, \\
z_{n} & =\inf \left\{s: s \geq b+1, \frac{l(s)}{s^{2}} \leq \frac{x_{n}^{2}}{n}\right\} .
\end{aligned}
$$

By an elementary argument and the assumption that $x_{n}^{2}=o(n)$, it is plain to see that

$$
z_{n} \rightarrow \infty \text { and } n l\left(z_{n}\right)=x_{n}^{2} z_{n}^{2} \text { for every } n \text { sufficiently large. }
$$


Since $E X^{2} I\{|X| \leq x\}$ is slowly varying,

$$
P(|X| \geq x)=o\left(l(x) / x^{2}\right), E|X| I\{|X| \geq x\}=o(l(x) / x)
$$

and

$$
E|X|^{k} I\{|X| \leq x\}=o\left(x^{k-2} l(x)\right) \text { for each } k>2
$$

as $x \rightarrow \infty$. For any $0<\varepsilon<1 / 2$, we have

$$
\begin{aligned}
& P\left(\frac{S_{n}}{V_{n}} \geq x_{n}\right) \leq P\left(\frac{S_{n}}{V_{n}} \geq x_{n}, V_{n}>0\right)+P\left(V_{n}=0\right) \\
& \leq P\left(\sum_{i=1}^{n} X_{i} I\left\{\left|X_{i}\right| \leq z_{n}\right\} \geq(1-\varepsilon) x_{n} V_{n}\right) \\
& +P\left(\sum_{i=1}^{n} X_{i} I\left\{\left|X_{i}\right|>z_{n}\right\} \geq \varepsilon x_{n} V_{n}, V_{n}>0\right)+P(X=0)^{n} \\
& \leq P\left(\sum_{i=1}^{n} X_{i} I\left\{\left|X_{i}\right| \leq z_{n}\right\} \geq(1-\varepsilon) x_{n}\left\{\sum_{i=1}^{n} X_{i}^{2} I\left\{\left|X_{i}\right| \leq z_{n}\right\}\right\}^{1 / 2}\right) \\
& +P\left(\sum_{i=1}^{n} I\left\{\left|X_{i}\right|>z_{n}\right\} \geq \varepsilon^{2} x_{n}^{2}\right)+P(X=0)^{n} \\
& \leq P\left(\sum_{i=1}^{n} X_{i} I\left\{\left|X_{i}\right| \leq z_{n}\right\} \geq(1-\varepsilon)^{2} x_{n} \sqrt{n l\left(z_{n}\right)}\right) \\
& +P\left(\sum_{i=1}^{n} X_{i}^{2} I\left\{\left|X_{i}\right| \leq z_{n}\right\} \leq(1-\varepsilon) n l\left(z_{n}\right)\right) \\
& +P\left(\sum_{i=1}^{n} I\left\{\left|X_{i}\right|>z_{n}\right\} \geq \varepsilon^{2} x_{n}^{2}\right)+P(X=0)^{n} \\
& :=J_{1}+J_{2}+J_{3}+P(X=0)^{n} \text {. }
\end{aligned}
$$

From the elementary inequalities

$$
\forall x \in R^{1}, \quad e^{x} \leq 1+x+\frac{x^{2}}{2}+\frac{|x|^{3}}{6} e^{x} \quad \text { and } \quad e^{x} \leq 1+x+\frac{x^{2}}{2} e^{|x|},
$$

it follows that for arbitrary bounded random variable $\xi$,

$$
E e^{\xi} \leq 1+E \xi+\frac{E \xi^{2}}{2}+\frac{E|\xi|^{3} e^{\xi}}{6}
$$

and

$$
E e^{\xi} \leq 1+E \xi+\frac{E \xi^{2} e^{|\xi|}}{2}
$$


By (4.4), (4.5), (4.8) and (4.6),

$$
\begin{aligned}
J_{1} & \leq \exp \left(-\frac{1}{z_{n}}(1-\varepsilon)^{2} x_{n} \sqrt{n l\left(z_{n}\right)}\right) E \exp \left(\frac{1}{z_{n}} \sum_{i=1}^{n} X_{i} I\left\{\left|X_{i}\right| \leq z_{n}\right\}\right) \\
& =\exp \left(-(1-\varepsilon)^{2} x_{n}^{2}\right)\left(E \exp \left(\frac{1}{z_{n}} X I\left\{|X| \leq z_{n}\right\}\right)\right)^{n} \\
& \leq \exp \left(-(1-\varepsilon)^{2} x_{n}^{2}\right)\left(1+\frac{E X I\left\{|X| \leq z_{n}\right\}}{z_{n}}+\frac{E X^{2} I\left\{|X| \leq z_{n}\right\}}{2 z_{n}^{2}}\right. \\
& =\exp \left(-(1-\varepsilon)^{2} x_{n}^{2}\right)\left(1-\frac{E X I\left\{|X|>z_{n}\right\}}{z_{n} I\left\{|X| \leq z_{n}\right\}}\right)^{n}+\frac{l\left(z_{n}\right)}{2 z_{n}^{2}} \\
& \leq \exp \left(-(1-\varepsilon)^{2} x_{n}^{2}\right)\left(1+\frac{l\left(z_{n}\right)}{2 z_{n}^{2}}+o\left(\frac{l\left(z_{n}\right)}{z_{n}^{2}}\right)\right)^{n} \\
& \leq \exp \left(-(1-\varepsilon)^{2} x_{n}^{2}\right) \exp \left(\frac{n l\left(z_{n}\right)}{2 z_{n}^{2}}+o\left(n \frac{l\left(z_{n}\right)}{z_{n}^{2}}\right)\right)^{3} I\left\{|X| \leq z_{n}\right\} \\
& =\exp \left(-(1-\varepsilon)^{2} x_{n}^{2}\right) \exp \left(\frac{x_{n}^{2}}{2}+o\left(x_{n}^{2}\right)\right) \\
& =\exp \left(-\left((1-\varepsilon)^{2}-\frac{1}{2}\right) x_{n}^{2}+o\left(x_{n}^{2}\right)\right) .
\end{aligned}
$$

As for $J_{2}$, similar to the proof of (4.10), using (4.9) instead of (4.8), we get

$$
\begin{aligned}
J_{2} & =P\left(\sum_{i=1}^{n} E X_{i}^{2} I\left\{\left|X_{i}\right| \leq z_{n}\right\}-X_{i}^{2} I\left\{\left|X_{i}\right| \leq z_{n}\right\} \geq \varepsilon n l\left(z_{n}\right)\right) \\
& \leq \exp \left(-n l\left(z_{n}\right) / z_{n}^{2}\right) E \exp \left(\frac{1}{\varepsilon z_{n}^{2}} \sum_{i=1}^{n} E X_{i}^{2} I\left\{\left|X_{i}\right| \leq z_{n}\right\}-X_{i}^{2} I\left\{\left|X_{i}\right| \leq z_{n}\right\}\right) \\
& =\exp \left(-x_{n}^{2}\right)\left(E \exp \left(\frac{1}{\varepsilon z_{n}^{2}}\left(E X^{2} I\left\{|X| \leq z_{n}\right\}-X^{2} I\left\{|X| \leq z_{n}\right\}\right)\right)\right)^{n} \\
& \leq \exp \left(-x_{n}^{2}\right)\left(1+\frac{E X^{4} I\left\{|X| \leq z_{n}\right\}}{2 \varepsilon^{2} z_{n}^{4}} \exp (1 / \varepsilon)\right)^{n} \\
& \leq \exp \left(-x_{n}^{2}\right)\left(1+\frac{\exp (1 / \varepsilon)}{\varepsilon^{2}} o\left(l\left(z_{n}\right) / z_{n}^{2}\right)\right)^{n} \\
& \leq \exp \left(-x_{n}^{2}\right) \exp \left(o\left(n l\left(z_{n}\right) / z_{n}^{2}\right)\right) \\
& =\exp \left(-x_{n}^{2}+o\left(x_{n}^{2}\right)\right) .
\end{aligned}
$$


We next estimate $J_{3}$. Recalling that $\sum_{i=1}^{n} I\left\{\left|X_{i}\right|>z_{n}\right\}$ has a binomial distribution and applying (2.8) again, we obtain from (4.5) and (4.4) that

$$
\begin{aligned}
J_{3} & \leq\left(\frac{3 n P\left(|X|>z_{n}\right)}{\varepsilon^{2} x_{n}^{2}}\right)^{\varepsilon^{2} x_{n}^{2}} \\
& =\left(o\left(\frac{l\left(z_{n}\right)}{z_{n}^{2}}\right) \frac{n}{\varepsilon^{2} x_{n}^{2}}\right)^{\varepsilon^{2} x_{n}^{2}}=\left(\frac{o(1)}{\varepsilon^{2}}\right)^{\varepsilon^{2} x_{n}^{2}} .
\end{aligned}
$$

Now (4.1) follows from the above inequalities and the arbitrariness of $\varepsilon$.

To prove (4.2), we need the following two lemmas.

LEMMA 4.1. Let $\left\{\xi, \xi_{n}, n \geq 1\right\}$ be a sequence of independent random variables, having the same nondegenerate distribution function $F(x)$. Assume that

$$
H:=\sup \left\{h: E e^{h \xi}<\infty\right\}>0 .
$$

For $0<h<H$, put

$$
m(h)=E \xi e^{h \xi} / E e^{h \xi}, \quad \sigma^{2}(h)=E \xi^{2} e^{h \xi} / E e^{h \xi}-m^{2}(h) .
$$

Then

$$
P\left(\sum_{i=1}^{n} \xi_{i} \geq n x\right) \geq \frac{3}{4}(E \exp (h \xi))^{n} \exp (-n h m(h)-2 h \sigma(h) \sqrt{n})
$$

provided that

$$
0<h<H \quad \text { and } \quad m(h) \geq x+2 \sigma(h) / \sqrt{n} .
$$

Proof. Let

$$
V(x)=\frac{1}{E e^{h \xi}} \int_{-\infty}^{x} e^{h y} d F(y) .
$$

Consider the sequence of independent random variables $\left\{\eta, \eta_{n}, n \geq 1\right\}$, having the same distribution function $V(x)$. Denote by $F_{n}(x)$ the distribution function of the random variable $\left(\sum_{i=1}^{n}\left(\eta_{i}-E \eta_{i}\right)\right) / \sqrt{n \operatorname{Var} \eta}$. In terms of the conjugate method [cf. (4.9) of Petrov (1965)], we have

$$
P\left(\sum_{i=1}^{n} \xi_{i} \geq n x\right)=\left(E e^{h \xi}\right)^{n} e^{-n h m(h)} \int_{-(m(h)-x) \sqrt{n} / \sigma(h)}^{\infty} e^{-h \sigma(h) t \sqrt{n}} d F_{n}(t) .
$$

By (4.12) and the Chebyshev inequality,

$$
\begin{aligned}
\int_{-(m(h)-x) \sqrt{n} / \sigma(h)}^{\infty} e^{-h \sigma(h) t \sqrt{n}} d F_{n}(t) & \geq \int_{-2}^{2} e^{-h \sigma(h) t \sqrt{n}} d F_{n}(t) \\
& \geq e^{-2 h \sigma(h) \sqrt{n}} P\left(\left|\sum_{i=1}^{n}\left(\eta_{i}-E \eta_{i}\right)\right| \leq 2 \sqrt{n \operatorname{Var} \eta}\right) \\
& \geq \frac{3}{4} e^{-2 h \sigma(h) \sqrt{n}} .
\end{aligned}
$$

This reduces to (4.11). 
LEMMA 4.2. Let

$0<\varepsilon<1 / 2, \quad b_{n}=1 / z_{n}, \quad \xi:=\xi_{n}=2 b_{n} X-b_{n}^{2} X^{2}, \quad h:=h_{\varepsilon}=(1+\varepsilon) / 2$, where $z_{n}$ is defined as in (4.3). Then, under the condition of Theorem 3.1,

$$
E e^{h \xi}=1+\varepsilon(1+\varepsilon) x_{n}^{2} /(2 n)+o\left(x_{n}^{2} / n\right),
$$

and

$$
E \xi e^{h \xi}=(1+2 \varepsilon) x_{n}^{2} / n+o\left(x_{n}^{2} / n\right)
$$

as $n \rightarrow \infty$.

Proof. Note that

$$
h \xi=h\left(1-\left(b_{n} X-1\right)^{2}\right) \leq h \leq 1 .
$$

In terms of (4.5), we have

$$
\begin{aligned}
E e^{h \xi}= & E e^{h \xi} I\left\{|X|>z_{n}\right\}+E e^{h \xi} I\left\{|X| \leq z_{n}\right\} \\
= & o\left(l\left(z_{n}\right) / z_{n}^{2}\right)+E\left(1+h \xi+\frac{(h \xi)^{2}}{2}\right) I\left\{|X| \leq z_{n}\right\} \\
& +E\left(e^{h \xi}-1-h \xi-\frac{(h \xi)^{2}}{2}\right) I\left\{|X| \leq z_{n}\right\} .
\end{aligned}
$$

From (4.4) to (4.6) it follows that

$$
\begin{aligned}
E(1+ & \left.h \xi+\frac{(h \xi)^{2}}{2}\right) I\left\{|X| \leq z_{n}\right\} \\
= & 1-P\left(|X|>z_{n}\right)-2 h b_{n} E X I\left\{|X|>z_{n}\right\}-h b_{n}^{2} l\left(z_{n}\right) \\
& +2 h^{2} b_{n}^{2} l\left(z_{n}\right)-2 h^{2} b_{n}^{3} E X^{3} I\left\{|X| \leq z_{n}\right\} \\
& +\frac{h^{2} b_{n}^{4} E X^{4} I\left\{|X| \leq z_{n}\right\}}{2} \\
= & 1-h b_{n}^{2} l\left(z_{n}\right)+2 h^{2} b_{n}^{2} l\left(z_{n}\right)+o\left(\frac{l\left(z_{n}\right)}{z_{n}^{2}}\right)+h b_{n} o\left(\frac{l\left(z_{n}\right)}{z_{n}}\right) \\
& +h^{2} b_{n}^{3} o\left(z_{n} l\left(z_{n}\right)\right)+h^{2} b_{n}^{4} o\left(z_{n}^{2} l\left(z_{n}\right)\right) \\
= & 1+\varepsilon(1+\varepsilon) b_{n}^{2} l\left(z_{n}\right) / 2+o\left(b_{n}^{2} l\left(z_{n}\right)\right) \\
= & 1+\varepsilon(1+\varepsilon) x_{n}^{2} /(2 n)+o\left(x_{n}^{2} / n\right) .
\end{aligned}
$$


Similarly, by using the inequality $\left|e^{x}-1-x-x^{2} / 2\right| \leq|x|^{3} e^{|x|}$,

$$
\begin{aligned}
& \left|E\left(\exp (h \xi)-1-h \xi-\frac{(h \xi)^{2}}{2}\right) I\left\{|X| \leq z_{n}\right\}\right| \\
& \quad \leq E|h \xi|^{3} \exp (h|\xi|) I\left\{|X| \leq z_{n}\right\} \\
& \quad \leq 4 h^{3} E \exp \left(h\left(1+\left(b_{n} X-1\right)^{2}\right)\right)\left(8 b_{n}^{3}|X|^{3}+b_{n}^{6} X^{6}\right) I\left\{|X| \leq z_{n}\right\} \\
& \quad \leq 4 h^{3} \exp 3 E\left(8 b_{n}^{3}|X|^{3}+b_{n}^{6} X^{6}\right) I\left\{|X| \leq z_{n}\right\} \\
& \quad \leq 4 h^{3} \exp 3\left(b_{n}^{3} o\left(z_{n} l\left(z_{n}\right)\right)+b_{n}^{6} o\left(z_{n}^{4} l\left(z_{n}\right)\right)\right) \\
& \quad=o\left(b_{n}^{2} l\left(z_{n}\right)\right)=o\left(x_{n}^{2} / n\right) .
\end{aligned}
$$

This proves (4.13), by (4.17), (4.18) and (4.19).

To estimate $E \xi e^{h \xi}$, write

$$
\begin{aligned}
E \xi e^{h \xi}= & E \xi e^{h \xi} I\left\{|X|>z_{n}\right\}+E \xi(1+h \xi) I\left\{|X| \leq z_{n}\right\} \\
& +E \xi\left(e^{h \xi}-1-h \xi\right) I\left\{|X| \leq z_{n}\right\} .
\end{aligned}
$$

Noting that $\sup _{-\infty<x \leq 1}|x| e^{x}=e$, we have

$$
\begin{aligned}
\left|E \xi e^{h \xi} I\left\{|X|>z_{n}\right\}\right| & \leq h^{-1} E h|\xi| e^{h \xi} I\left\{|X|>z_{n}\right\} \\
& \leq h^{-1} \text { eP }\left(|X|>z_{n}\right) \\
& =h^{-1} o\left(l\left(z_{n}\right) / z_{n}^{2}\right) \\
& =o\left(x_{n}^{2} / n\right)
\end{aligned}
$$

by (4.16) and (4.5). Similar to (4.18),

$$
E \xi(1+h \xi) I\left\{|X| \leq z_{n}\right\}=(1+2 \varepsilon) x_{n}^{2} / n+o\left(x_{n}^{2} / n\right) .
$$

In terms of the inequality that $\left|e^{x}-1-x\right| \leq x^{2} e^{|x|}$, along the lines of the proof of (4.19), one can get

$$
E \xi\left(e^{h \xi}-1-h \xi\right) I\left\{|X| \leq z_{n}\right\}=o\left(x_{n}^{2} / n\right) .
$$

This reduces to (4.14). The proof of (4.15) is similar to that of (4.14) and so is omitted here.

We are now ready to prove (4.2). Let $b_{n}, h$ and $\xi$ be defined as in Lemma 4.2. Put

$$
\xi_{i}=2 b_{n} X_{i}-b_{n}^{2} X_{i}^{2}, \quad i=1,2, \ldots
$$

By (2.2) we have

$$
\begin{aligned}
P\left(\frac{S_{n}}{V_{n}} \geq x_{n}\right) & \geq P\left(S_{n} \geq \frac{1}{2 b_{n}}\left(b_{n}^{2} V_{n}^{2}+x_{n}^{2}\right)\right) \\
& =P\left(\sum_{i=1}^{n} \xi_{i} \geq x_{n}^{2}\right) .
\end{aligned}
$$


Let

$$
m(h)=E \xi e^{h \xi} / E e^{h \xi}, \quad \sigma^{2}(h)=E \xi^{2} e^{h \xi} / E e^{h \xi}-m^{2}(h) \quad \text { and } \quad x=x_{n}^{2} / n
$$

in Lemma 4.1. From Lemma 4.2 it is clear that

$$
\begin{gathered}
m(h)=(1+2 \varepsilon) x_{n}^{2} / n+o\left(x_{n}^{2} / n\right), \\
E \xi e^{h \xi}-\left(x_{n}^{2} / n\right) E e^{h \xi}=2 \varepsilon x_{n}^{2} / n+o\left(x_{n}^{2} / n\right)
\end{gathered}
$$

and

$$
\frac{\sigma(h)\left(E e^{h \xi}\right)^{1 / 2}}{\sqrt{n}}=\frac{2(1+o(1)) x_{n} / \sqrt{n}}{\sqrt{n}}=o\left(\frac{x_{n}^{2}}{n}\right) .
$$

Therefore, (4.12) is satisfied for every sufficiently large $n$. By Lemma 4.1 and (4.13),

$$
\begin{aligned}
P\left(\sum_{i=1}^{n} \xi_{i} \geq x_{n}^{2}\right) & \geq \frac{3}{4}(E \exp (h \xi))^{n} \exp (-n h m(h)-2 h \sigma(h) \sqrt{n}) \\
& \geq \frac{3}{4} \exp \left(\varepsilon(1+\varepsilon) x_{n}^{2} / 2-h(1+2 \varepsilon) x_{n}^{2}+o\left(x_{n}^{2}\right)\right) \\
& =\frac{3}{4} \exp \left(-(1+\varepsilon)^{2} x_{n}^{2} / 2+o\left(x_{n}^{2}\right)\right) .
\end{aligned}
$$

This proves (4.2) by (4.20) and (4.21) and the arbitrariness of $\varepsilon$. The proof of Theorem 3.1 is now complete.

REMARK 4.1. Along with the above proof we have actually proved that the convergence in (3.1) is uniform: for arbitrary $0<\varepsilon<1 / 2$, there exist $0<\delta<$ $1, x_{0}>1$ and $n_{0}$ such that for any $n \geq n_{0}$ and $x_{0}<x<\delta \sqrt{n}$,

$$
\exp \left(-(1+\varepsilon) x^{2} / 2\right) \leq P\left(\frac{S_{n}}{V_{n}} \geq x\right) \leq \exp \left(-(1-\varepsilon) x^{2} / 2\right)
$$

REMARK 4.2. By using the Ottaviani maximum inequality and according to the above proof, one can obtain that under the condition of Theorem 3.1, for any $0<\varepsilon<1$, there exist $0<\delta<1, x_{0}>1$ and $n_{0}$ such that for any $n \geq n_{0}$ and $x_{0}<x<\delta \sqrt{n}$,

$$
P\left(\max _{n / 2 \leq k \leq n} \frac{S_{k}}{V_{k}} \geq x\right) \leq \exp \left(\frac{-(1-\varepsilon) x^{2}}{2}\right) .
$$

To prove Theorem 3.3, we start with some preliminary lemmas. For the sake of convenience, statements below are understood to hold for every sufficiently large $n$. Let

$$
q=p /(p-1), \quad y_{n}=x_{n}^{q} / n
$$

and let $z_{n}$ be a sequence of positive numbers such that

$$
h\left(z_{n}\right) z_{n}^{-\alpha} \sim y_{n} \text { as } n \rightarrow \infty .
$$


LEMMA 4.3. Under the conditions of Theorem 3.3, we have

$$
E|X|{ }^{p} I\{|X| \leq x\} \sim \frac{\alpha\left(c_{1}+c_{2}\right)}{p-\alpha} x^{p-\alpha} h(x),
$$

$$
E|X| I\{|X| \geq x\} \sim \frac{\alpha\left(c_{1}+c_{2}\right)}{\alpha-1} x^{1-\alpha} h(x) \text { if } 1<\alpha<2,
$$

$$
E|X| I\{|X| \leq x\} \sim \frac{\alpha\left(c_{1}+c_{2}\right)}{1-\alpha} x^{1-\alpha} h(x) \quad \text { if } 0<\alpha<1,
$$

as $x \rightarrow \infty$.

The proofs are straightforward and so are omitted here.

Lemma 4.4. Let $2^{p}<D<\infty$. Under the conditions of Theorem 3.3, for any $2^{p} \leq t \leq D$ and every sufficiently large $n$

$$
E \exp \left(-t z_{n}^{-p}|X|^{p}\right) \leq \exp \left(-\left(c_{1}+c_{2}\right) t^{\alpha /(2 p)} y_{n} / 150\right) .
$$

Proof. Integration by parts leads to

$$
\begin{aligned}
1-E \exp \left(-t z_{n}^{-p}|X|^{p}\right) & =p \int_{0}^{\infty} x^{p-1} \exp \left(-x^{p}\right) P\left(|X| \geq x z_{n} t^{-1 / p}\right) d x \\
& \geq p \int_{1}^{t^{1 / p}} x^{p-1} \exp \left(-x^{p}\right) P\left(|X| \geq x z_{n} t^{-1 / p}\right) d x .
\end{aligned}
$$

By (4.27), (H4) and (4.23),

$$
\begin{aligned}
p \int_{1}^{t^{1 / p}} & x^{p-1} \exp \left(-x^{p}\right) P\left(|X| \geq x z_{n} t^{-1 / p}\right) d x \\
& \geq(1 / 2) p\left(c_{1}+c_{2}\right) \int_{1}^{t^{1 / p}} x^{p-1} \exp \left(-x^{p}\right)\left(x z_{n} t^{-1 / p}\right)^{-\alpha} h\left(x z_{n} t^{-1 / p}\right) d x \\
& =(1 / 2) p\left(c_{1}+c_{2}\right) t^{\alpha / p} z_{n}^{-\alpha} \int_{1}^{t^{1 / p}} x^{p-1-\alpha} h\left(x z_{n} t^{-1 / p}\right) \exp \left(-x^{p}\right) d x \\
& \geq(1 / 2) p\left(c_{1}+c_{2}\right) t^{\alpha / p} z_{n}^{-\alpha} h\left(z_{n} t^{-1 / p}\right) \int_{1}^{2} x^{p-1-2 \alpha} \exp \left(-x^{p}\right) d x \\
& \geq(1 / 32)\left(c_{1}+c_{2}\right) t^{\alpha / p} z_{n}^{-\alpha} h\left(z_{n} t^{-1 / p}\right) \int_{1}^{2} p x^{p-1} \exp \left(-x^{p}\right) d x \\
& \geq\left(c_{1}+c_{2}\right) t^{\alpha / p} z_{n}^{-\alpha} h\left(z_{n} t^{-1 / p}\right) / 140 \\
& \geq\left(c_{1}+c_{2}\right) t^{\alpha /(2 p)} z_{n}^{-\alpha} h\left(z_{n}\right) / 145 \\
& \geq\left(c_{1}+c_{2}\right) t^{\alpha /(2 p)} y_{n} / 150 .
\end{aligned}
$$

Now (4.28) follows from the above inequalities. 
For $t>0$, put

$$
\gamma(t)= \begin{cases}c_{1} \alpha \int_{0}^{\infty} \frac{1+p t x-\exp \left(t\left(p x-x^{p}\right)\right)}{x^{\alpha+1}} d x & \\ +c_{2} \alpha \int_{0}^{\infty} \frac{1-p t x-\exp \left(t\left(-p x-x^{p}\right)\right)}{x^{\alpha+1}} d x, & \text { if } 1<\alpha<2, \\ c_{1} \int_{0}^{\infty} \frac{2-\exp \left(t\left(p x-x^{p}\right)\right)-\exp \left(t\left(-p x-x^{p}\right)\right)}{x^{2}} d x, & \text { if } \alpha=1, \\ c_{1} \alpha \int_{0}^{\infty} \frac{1-\exp \left(t\left(p x-x^{p}\right)\right)}{x^{\alpha+1}} d x & \text { if } 0<\alpha<1 . \\ +c_{2} \alpha \int_{0}^{\infty} \frac{1-\exp \left(t\left(-p x-x^{p}\right)\right)}{x^{\alpha+1}} d x, & \end{cases}
$$

Clearly, we have

$$
\gamma^{\prime}(t)=\left\{\begin{array}{cc}
c_{1} \alpha \int_{0}^{\infty} \frac{p-\left(p-x^{p-1}\right) \exp \left(t\left(p x-x^{p}\right)\right)}{x^{\alpha}} d x & \text { if } 1<\alpha<2, \\
+c_{2} \alpha \int_{0}^{\infty} \frac{\left(p+x^{p-1}\right) \exp \left(t\left(-p x-x^{p}\right)\right)-p}{x^{\alpha}} d x & \\
c_{1} \int_{0}^{\infty}\left[\frac{\left(x^{p-1}-p\right) \exp \left(t\left(p x-x^{p}\right)\right)}{x}\right. & \text { if } \alpha=1, \\
\left.+\frac{\left(p+x^{p-1}\right) \exp \left(t\left(-p x-x^{p}\right)\right)}{x}\right] d x, & \\
c_{1} \alpha \int_{0}^{\infty} \frac{\left(x^{p-1}-p\right) \exp \left(t\left(p x-x^{p}\right)\right)}{x^{\alpha}} d x & \text { if } 0<\alpha<1 \\
+c_{2} \alpha \int_{0}^{\infty} \frac{\left(p+x^{p-1}\right) \exp \left(t\left(-p x-x^{p}\right)\right)}{x^{\alpha}} d x, &
\end{array}\right.
$$

and

$$
\text { (4.31) } \gamma^{\prime \prime}(t)=\left\{\begin{array}{cc}
-c_{1} \alpha \int_{0}^{\infty} \frac{\left(p-x^{p-1}\right)^{2} \exp \left(t\left(p x-x^{p}\right)\right)}{x^{\alpha-1}} d x & \\
-c_{2} \alpha \int_{0}^{\infty} \frac{\left(p+x^{p-1}\right)^{2} \exp \left(t\left(-p x-x^{p}\right)\right)}{x^{\alpha-1}} d x, & \text { if } 1<\alpha<2, \\
-c_{1} \int_{0}^{\infty}\left[\left(x^{p-1}-p\right)^{2} \exp \left(t\left(p x-x^{p}\right)\right)\right. & \\
\left.+(2+x)^{2} \exp \left(t\left(-p x-x^{p}\right)\right)\right] d x, & \text { if } \alpha=1, \\
-c_{1} \alpha \int_{0}^{\infty} \frac{\left(x^{p-1}-p\right)^{2} \exp \left(t\left(p x-x^{p}\right)\right)}{x^{\alpha-1}} d x & \\
-c_{2} \alpha \int_{0}^{\infty} \frac{\left(p+x^{p-1}\right)^{2} \exp \left(t\left(-p x-x^{p}\right)\right)}{x^{\alpha}} d x, & \text { if } 0<\alpha<1 .
\end{array}\right.
$$

The next two lemmas play a key role in the proof of Theorem 3.3. 
LEMMA 4.5. Let

$$
\xi:=\xi_{b}=p b X-|b X|^{p}, \quad b>0
$$

and let $0<d<D<\infty$. Under the conditions of Theorem 3.3, as $b \downarrow 0$,

$$
\begin{gathered}
1-E e^{t \xi}=\gamma(t) b^{\alpha} h(1 / b)+o\left(b^{\alpha} h(1 / b)\right), \\
E \xi e^{t \xi}=-b^{\alpha} h(1 / b) \gamma^{\prime}(t)+o\left(b^{\alpha} h(1 / b)\right)
\end{gathered}
$$

and

$$
E \xi^{2} e^{t \xi}=-b^{\alpha} h(1 / b) \gamma^{\prime \prime}(t)+o\left(b^{\alpha} h(1 / b)\right)
$$

for any $d \leq t \leq D$, where $\gamma(t)$ is defined as in (4.29) and the constants implied in $o(\cdot)$ do not depend on $t$.

Proof. We divide the proof into three different cases.

Case 1. $1<\alpha<2$. Since $E X=0$,

$$
\begin{aligned}
1-E e^{t \xi}= & p t \int_{0}^{\infty} P(X \geq x / b)\left(1-\left(1-x^{p-1}\right) \exp \left(t\left(p x-x^{p}\right)\right)\right) d x \\
& +p t \int_{0}^{\infty} P(X \leq-x / b)\left(-1+\left(1+x^{p-1}\right) \exp \left(t\left(-p x-x^{p}\right)\right)\right) d x \\
:= & I_{1}+I_{2} .
\end{aligned}
$$

Let $\theta=(1+\alpha \max (1 / 2,1 / p)) / 2$. Then

$$
\alpha \max (1 / 2,1 / p)<\theta<1
$$

and

$$
\begin{aligned}
I_{1}= & p t \int_{0}^{b^{\theta}} P(X \geq x / b)\left(1-\left(1-x^{p-1}\right) \exp \left(t\left(p x-x^{p}\right)\right)\right) d x \\
& \quad+p t \int_{b^{\theta}}^{\infty} P(X \geq x / b)\left(1-\left(1-x^{p-1}\right) \exp \left(t\left(p x-x^{p}\right)\right)\right) d x \\
:= & I_{1,1}+I_{1,2} .
\end{aligned}
$$

It is easy to see that for $t>0$ and $x>0$,

$$
\begin{aligned}
\mid 1- & \left(1-x^{p-1}\right) \exp \left(t\left(p x-x^{p}\right)\right) \mid \\
\leq & \left|1-\exp \left(t\left(p x-x^{p}\right)\right)\right|+x^{p-1} \exp \left(t\left(p x-x^{p}\right)\right) \\
\leq & \min \left(1+\exp (t(p-1)), x K_{p} \exp (t p)\right) \\
& \quad+\min \left(x^{p-1} \exp (t(p-1)), K_{p}\left(1+t^{1-p}\right) \exp (t(p-1))\right) \\
\leq & K_{p}\left(1+t^{1-p}\right) \exp (t p) \min \left(1, x+x^{p-1}\right)
\end{aligned}
$$


for some constant $K_{p}$ depending only on $p$. From (4.36) we obtain

$$
\begin{aligned}
I_{1,1} & \leq p t \int_{0}^{b^{\theta}} K_{p} e^{t p}\left(1+t^{1-p}\right)\left(x+x^{p-1}\right) d x \\
& \leq p K_{p} D\left(1+d^{1-p}\right) e^{D p}\left(b^{2 \theta}+b^{p \theta}\right)=o\left(b^{\alpha} h(1 / b)\right) .
\end{aligned}
$$

In terms of (3.2), (4.36) and ( $\mathrm{H} 4)$, we get

$$
\begin{aligned}
I_{1,2}= & p t \int_{b^{\theta}}^{\infty} \frac{c_{1} b^{\alpha} h(x / b)}{x^{\alpha}}\left(1-\left(1-x^{p-1}\right) \exp \left(t\left(p x-x^{p}\right)\right)\right) d x \\
& +o(1) \int_{b^{\theta}}^{\infty} \frac{c_{1} b^{\alpha} h(x / b)}{x^{\alpha}}\left|1-\left(1-x^{p-1}\right) \exp \left(t\left(p x-x^{p}\right)\right)\right| d x \\
= & p t c_{1} b^{\alpha} h\left(\frac{1}{b}\right) \int_{b^{\theta}}^{\infty} \frac{1-\left(1-x^{p-1}\right) \exp \left(t\left(p x-x^{p}\right)\right)}{x^{\alpha}} d x \\
& +p t c_{1} b^{\alpha} h\left(\frac{1}{b}\right) \int_{b^{\theta}}^{\infty}\left(\frac{h(x / b)}{h(1 / b)}-1\right) \frac{1-\left(1-x^{p-1}\right) \exp \left(t\left(p x-x^{p}\right)\right)}{x^{\alpha}} d x \\
& +o(1) b^{\alpha} h\left(\frac{1}{b}\right)\left(1+t^{1-p}\right) \exp (t p) \int_{b^{\theta}}^{\infty} \frac{h(x / b) \min \left(1, x+x^{p-1}\right)}{h(1 / b) x^{\alpha}} d x \\
= & p t c_{1} b^{\alpha} h\left(\frac{1}{b}\right) \int_{0}^{\infty} \frac{1-\left(1-x^{p-1}\right) \exp \left(t\left(p x-x^{p}\right)\right)}{x^{\alpha}} d x+o(1) b^{\alpha} h\left(\frac{1}{b}\right) \\
& +p t c_{1} b^{\alpha} h\left(\frac{1}{b}\right) \int_{b^{\theta}}^{\infty}\left(\frac{h(x / b)}{h(1 / b)}-1\right) \frac{1-\left(1-x^{p-1}\right) \exp \left(t\left(p x-x^{p}\right)\right)}{x^{\alpha}} d x \\
& +o(1) b^{\alpha} h\left(\frac{1}{b}\right)\left(1+t^{1-p}\right) \exp (t p) \\
& \times \int_{b^{\theta}}^{\infty}\left(x+\frac{1}{x}\right) \\
= & \alpha c_{1} b^{\alpha} h\left(\frac{1}{b}\right) \int_{0}^{\infty} \frac{1+p t x-\exp \left(t\left(p x-x^{p}\right)\right)}{x^{\alpha+1}} d x+o(1) b^{\alpha} h\left(\frac{1}{b}\right) \\
& +p t c_{1} b^{\alpha} h\left(\frac{1}{b}\right) \int_{b^{\theta}}^{\infty}\left(\frac{h(x / b)}{h(1 / b)}-1\right) \frac{1-\left(1-x^{p-1}\right) \exp \left(t\left(p x-x^{p}\right)\right)}{x^{\alpha}} d x,
\end{aligned}
$$

by an integration by parts. Use (H4) and (4.36) again, for any $0<\varepsilon<\min (2-$ $\alpha, \alpha-1, p-\alpha) / 2$,

$$
\begin{aligned}
\int_{b^{\theta}}^{\infty} & \frac{h(x / b)}{h(1 / b)}-1|| \frac{1-\left(1-x^{p-1}\right) \exp \left(t\left(p x-x^{p}\right)\right)}{x^{\alpha}} \mid d x \\
& \leq 2 K_{p}\left(1+t^{1-p}\right) \exp (t p) \int_{b^{\theta}}^{\infty}\left(\left(x \vee \frac{1}{x}\right)^{\varepsilon}-1\right) \frac{\min \left(1, x+x^{p-1}\right)}{x^{\alpha}} d x \\
& \leq 2 K_{p}\left(1+d^{1-p}\right) \exp (p D) \int_{0}^{\infty}\left(\left(x \vee \frac{1}{x}\right)^{\varepsilon}-1\right) \frac{\min \left(1, x+x^{p-1}\right)}{x^{\alpha}} d x
\end{aligned}
$$


Since

$$
\lim _{\varepsilon \downarrow 0} \int_{0}^{\infty}\left(\left(x \vee \frac{1}{x}\right)^{\varepsilon}-1\right) \frac{\min \left(1, x+x^{p-1}\right)}{x^{\alpha}} d x=0,
$$

the above inequalities yield

$$
I_{1}=\alpha c_{1} b^{\alpha} h\left(\frac{1}{b}\right) \int_{0}^{\infty} \frac{1+p t x-\exp \left(t\left(p x-x^{p}\right)\right)}{x^{\alpha+1}} d x+o(1) b^{\alpha} h\left(\frac{1}{b}\right)
$$

Similarly, we have

$$
I_{2}=\alpha c_{2} b^{\alpha} h\left(\frac{1}{b}\right) \int_{0}^{\infty} \frac{1-p t x-\exp \left(t\left(-p x-x^{p}\right)\right)}{x^{\alpha+1}} d x+o(1) b^{\alpha} h\left(\frac{1}{b}\right)
$$

This proves (4.32), by (4.37) and (4.38).

As for $E \xi e^{t \xi}$, notice that

$$
\begin{aligned}
& E \xi e^{t \xi}=p \int_{0}^{\infty} P(X \geq x / b)\left(\left(1-x^{p-1}\right)\left(t\left(p x-x^{p}\right)+1\right)\right.\left.\times \exp \left(t\left(p x-x^{p}\right)\right)-1\right) d x \\
&+p \int_{0}^{\infty} P(X \leq-x / b)\left(\left(1+x^{p-1}\right)\left(t\left(p x+x^{p}\right)-1\right)\right. \\
&\left.\times \exp \left(t\left(-p x-x^{p}\right)\right)+1\right) d x .
\end{aligned}
$$

Similar to (4.37) and (4.38), one can obtain that the right hand side of (4.39) is equal to

$$
\begin{aligned}
& p c_{1} b^{\alpha} h\left(\frac{1}{b}\right) \int_{0}^{\infty} \frac{\left(1-x^{p-1}\right)\left(t\left(p x-x^{p}\right)+1\right) \exp \left(t\left(p x-x^{p}\right)\right)-1}{x^{\alpha}} d x \\
& +p c_{2} b^{\alpha} h\left(\frac{1}{b}\right) \int_{0}^{\infty} \frac{\left(1+x^{p-1}\right)\left(t\left(p x+x^{p}\right)-1\right) \exp \left(t\left(-p x-x^{p}\right)\right)+1}{x^{\alpha}} d x \\
& +o\left(b^{\alpha} h\left(\frac{1}{b}\right)\right) \\
& =c_{1} b^{\alpha} h\left(\frac{1}{b}\right) \int_{0}^{\infty} \frac{1}{x^{\alpha}}\left(\left(p x-x^{p}\right) \exp \left(t\left(p x-x^{p}\right)\right)-p x\right)^{\prime} d x \\
& \quad+c_{2} b^{\alpha} h\left(\frac{1}{b}\right) \int_{0}^{\infty} \frac{1}{x^{\alpha}}\left(\left(-p x-x^{p}\right) \exp \left(t\left(-p x-x^{p}\right)\right)+p x\right)^{\prime} d x \\
& \quad+o\left(b^{\alpha} h\left(\frac{1}{b}\right)\right)
\end{aligned}
$$




$$
\begin{aligned}
= & \alpha c_{1} b^{\alpha} h\left(\frac{1}{b}\right) \int_{0}^{\infty} \frac{\left(p-x^{p-1}\right) \exp \left(t\left(p x-x^{p}\right)\right)-p}{x^{\alpha}} d x \\
& +\alpha c_{2} b^{\alpha} h\left(\frac{1}{b}\right) \int_{0}^{\infty} \frac{\left(-p-x^{p-1}\right) \exp \left(t\left(-p x-x^{p}\right)\right)+p}{x^{\alpha}} d x \\
& +o\left(b^{\alpha} h\left(\frac{1}{b}\right)\right) \\
= & -b^{\alpha} h\left(\frac{1}{b}\right) \gamma^{\prime}(t)+o\left(b^{\alpha} h\left(\frac{1}{b}\right)\right),
\end{aligned}
$$

as desired.

The proof of (4.34) is along the same lines as that of (4.32). One has

$$
\begin{aligned}
E \xi^{2} e^{t \xi}= & \int_{0}^{\infty} P\left(X \geq \frac{x}{b}\right)\left(\left(p x-x^{p}\right)^{2} \exp \left(t\left(p x-x^{p}\right)\right)\right)^{\prime} d x \\
& +\int_{0}^{\infty} P\left(X \leq \frac{-x}{b}\right)\left(\left(p x+x^{p}\right)^{2} \exp \left(t\left(-p x-x^{p}\right)\right)\right)^{\prime} d x \\
= & c_{1} b^{\alpha} h\left(\frac{1}{b}\right) \int_{0}^{\infty} x^{-\alpha}\left(\left(p x-x^{p}\right)^{2} \exp \left(t\left(p x-x^{p}\right)\right)\right)^{\prime} d x \\
& +c_{2} b^{\alpha} h\left(\frac{1}{b}\right) \int_{0}^{\infty} x^{-\alpha}\left(\left(p x+x^{p}\right)^{2} \exp \left(t\left(-p x-x^{p}\right)\right)\right)^{\prime} d x \\
& +o\left(b^{\alpha} h\left(\frac{1}{b}\right)\right) \\
= & \alpha c_{1} b^{\alpha} h\left(\frac{1}{b}\right) \int_{0}^{\infty} \frac{\left(p-x^{p-1}\right)^{2} \exp \left(t\left(p x-x^{p}\right)\right)}{x^{\alpha-1}} d x \\
& +\alpha c_{2} b^{\alpha} h\left(\frac{1}{b}\right) \int_{0}^{\infty} \frac{\left(p-x^{p-1}\right)^{2} \exp \left(t\left(p x-x^{p}\right)\right)}{x^{\alpha-1}} d x+o\left(b^{\alpha} h\left(\frac{1}{b}\right)\right) \\
= & -b^{\alpha} h\left(\frac{1}{b}\right) \gamma^{\prime \prime}(t)+o\left(b^{\alpha} h\left(\frac{1}{b}\right)\right),
\end{aligned}
$$

as desired.

Case 2. $\alpha=1$. Since $X$ is symmetric,

$$
\begin{aligned}
1-E \exp (t \xi)= & -\int_{0}^{\infty}\left(1-\exp \left(t\left(p x-x^{p}\right)\right)\right) d P(X \geq x / b) \\
& -\int_{0}^{\infty}\left(1-\exp \left(t\left(-p x-x^{p}\right)\right)\right) d P(X \leq-x / b) \\
= & -\int_{0}^{\infty}\left(2-\exp \left(t\left(p x-x^{p}\right)\right)-\exp \left(t\left(-p x-x^{p}\right)\right)\right) d P(X \geq x / b) \\
= & \int_{0}^{\infty} P(X \geq x / b)\left(2-\exp \left(t\left(p x-x^{p}\right)\right)-\exp \left(t\left(-p x-x^{p}\right)\right)\right)^{\prime} d x .
\end{aligned}
$$

The remainder of the proof is almost the same as in Case 1. 
The proofs of (4.33) and (4.34) are similar and so the details are omitted here.

Case 3. $0<\alpha<1$. In this case, note that

$$
\begin{aligned}
1-E e^{t \xi}= & -\int_{0}^{\infty}\left(1-\exp \left(t\left(p x-x^{p}\right)\right)\right) d P(X \geq x / b) \\
& -\int_{0}^{\infty}\left(1-\exp \left(t\left(-p x-x^{p}\right)\right)\right) d P(X \leq-x / b) \\
= & \int_{0}^{\infty} P(X \geq x / b)\left(1-\exp \left(t\left(p x-x^{p}\right)\right)\right)^{\prime} d x \\
& +\int_{0}^{\infty} P(X \geq x / b)\left(1-\exp \left(t\left(-p x-x^{p}\right)\right)\right)^{\prime} d x .
\end{aligned}
$$

The rest of the proof is along the same lines as in Case 1.

Lemma 4.6. Let $0<d \leq D<\infty$. Then, under the conditions of Theorem 3.3,

$$
\begin{aligned}
& \sup _{0<b \leq D / z_{n}} \inf _{t>0} \exp \left(-t c y_{n}\right) E \exp \left(t\left(p b X-|b X|^{p}\right)\right) \\
& \quad \leq \exp \left(-\beta_{p} c y_{n}+o\left(y_{n}\right)\right)
\end{aligned}
$$

for every $d \leq c \leq D$, where $\beta_{p}:=\beta_{p}\left(\alpha, c_{1}, c_{2}\right)$ is defined as in Theorem 3.3, $z_{n}$ and $y_{n}$ are as in (4.22) and (4.23) and the constant implied by $o\left(y_{n}\right)$ is uniform in $c \in[d, D]$.

Proof. Let $0<\delta<d$. From (4.32) it follows that for $0<b<\delta / z_{n}$,

$$
\begin{aligned}
E \exp \left(3 \beta_{p}\left(p b X-|b X|^{p}\right)\right) & \leq 1-\gamma\left(3 \beta_{p}\right) b^{\alpha} h(1 / b)+o\left(b^{\alpha} h(1 / b)\right) \\
& \leq \exp \left(\left(\left|\gamma\left(3 \beta_{p}\right)\right|+1\right) b^{\alpha} h(1 / b)\right) \\
& \leq \exp \left(K\left(\delta / z_{n}\right)^{\alpha} h\left(z_{n} / \delta\right)\right) \\
& \leq \exp \left(K \delta^{\alpha / 2} z_{n}^{-\alpha} h\left(z_{n}\right)\right) \\
& \leq \exp \left(K \delta^{\alpha / 2} y_{n}\right) \\
& \leq \exp \left(d \beta_{p} y_{n}\right) \\
& \leq \exp \left(c \beta_{p} y_{n}\right),
\end{aligned}
$$

provided that $\delta$ is chosen to be sufficiently small, and that $n$ is large enough; here and in the sequel $K$ and $K_{1}, K_{2}, \ldots$ denote positive constants which depend only on $\alpha, p$ and other given constants, but may be different from line to line. Therefore, there exists $\delta>0$ such that

$$
\begin{aligned}
& \sup _{0<b \leq \delta / z_{n}} \inf _{t>0} \exp \left(-t c y_{n}\right) E \exp \left(t\left(p b X-|b X|^{p}\right)\right) \\
& \quad \leq \sup _{0<b \leq \delta / z_{n}} \exp \left(-3 \beta_{p} c y_{n}\right) E \exp \left(3 \beta_{p}\left(p b X-|b X|^{p}\right)\right) \\
& \quad \leq \exp \left(-2 \beta_{p} c y_{n}\right) .
\end{aligned}
$$


Next estimate $\sup _{\delta / z_{n} \leq b \leq D / z_{n}}$ inf $_{t>0} \exp \left(-t c y_{n}\right) E \exp \left(t\left(p b X-|b X|^{p}\right)\right)$. Let $\gamma(t), \gamma^{\prime}(t)$ and $\gamma^{\prime \prime}(t)$ be defined as in (4.29), (4.30) and (4.31), respectively. In view of (4.31) and the fact that

$$
\gamma^{\prime \prime}(t)<0 \text { for } t>0, \quad \lim _{t \downarrow 0} \gamma^{\prime}(t)=\infty \quad \text { and } \quad \lim _{t \uparrow \infty} \gamma^{\prime}(t)=-\infty,
$$

there exists a unique $t_{b}$ such that

$$
\gamma^{\prime}\left(t_{b}\right)=-\frac{y_{n} c}{b^{\alpha} h\left(z_{n}\right)} .
$$

Since

$$
0<K_{1} \leq \frac{d y_{n} z_{n}^{\alpha}}{D^{\alpha} h\left(z_{n}\right)} \leq \frac{y_{n} c}{b^{\alpha} h\left(z_{n}\right)} \leq \frac{D y_{n} z_{n}^{\alpha}}{\delta^{\alpha} h\left(z_{n}\right)} \leq K_{2}<\infty
$$

for $\delta / z_{n} \leq b \leq D / z_{n}$, we have

$$
K_{3} \leq t_{b} \leq K_{4}
$$

Applying ( $\mathrm{H} 2)$ and (4.32) again, we obtain

$$
\begin{aligned}
\sup _{\delta / z_{n} \leq b \leq D / z_{n}} \inf _{t>0} \exp \left(-t c y_{n}\right) E \exp \left(t\left(p b X-|b X|^{p}\right)\right) \\
\quad \leq \sup _{\delta / z_{n} \leq b \leq D / z_{n}} \exp \left(-t_{b} c y_{n}\right) E \exp \left(t_{b}\left(p b X-|b X|^{p}\right)\right) \\
\quad \leq \sup _{\delta / z_{n} \leq b \leq D / z_{n}} \exp \left(-t_{b} c y_{n}-\gamma\left(t_{b}\right) b^{\alpha} h(1 / b)+o\left(b^{\alpha} h(1 / b)\right)\right) \\
\quad \leq \sup _{\delta / z_{n} \leq b \leq D / z_{n}} \exp \left(-t_{b} c y_{n}-\gamma\left(t_{b}\right) b^{\alpha} h\left(z_{n}\right)+\gamma\left(t_{b}\right) b^{\alpha} h\left(z_{n}\right) o(1)+o\left(y_{n}\right)\right) \\
\quad \leq \sup _{\delta / z_{n} \leq b \leq D / z_{n}} \exp \left(-t_{b} c y_{n}-\gamma\left(t_{b}\right) b^{\alpha} h\left(z_{n}\right)+o\left(y_{n}\right)\right) .
\end{aligned}
$$

Let

$$
g(b)=-t_{b} c y_{n}-\gamma\left(t_{b}\right) b^{\alpha} h\left(z_{n}\right)
$$

and $b_{0}$ be such that $t_{b_{0}}=\beta_{p}$. Noting that $\gamma(t)=\alpha t^{\alpha} \Gamma_{p}\left(t, \alpha, c_{1}, c_{2}\right)$, we have

$$
\gamma\left(t_{b_{0}}\right)=0 \text {. }
$$

By (4.43),

$$
g^{\prime}(b)=-\gamma\left(t_{b}\right) \alpha b^{\alpha-1} h\left(z_{n}\right) \begin{cases}>0, & \text { if } b<b_{0}, \\ =0, & \text { if } b=b_{0}, \\ <0, & \text { if } b>b_{0},\end{cases}
$$

for $t_{b}$ is a decreasing function of $b$, and $\gamma(t) / t^{\alpha}$ is a decreasing function of $t$. Thus, $g(b)$ achieves the maximum at $b=b_{0}$ and $g\left(b_{0}\right)=-\beta_{p} c y_{n}$. Consequently,

$$
\begin{aligned}
& \sup _{\delta / z_{n} \leq b \leq D / z_{n}} \inf _{t>0} \exp \left(-t c y_{n}\right) E \exp \left(t\left(p b X-|b X|^{p}\right)\right) \\
& \quad \leq \exp \left(-\beta_{p} c y_{n}+o\left(y_{n}\right)\right) .
\end{aligned}
$$

This proves (4.41) by (4.42) and (4.44). 
Proof of Theorem 3.3. Let $\beta_{p}=\beta_{p}\left(\alpha, c_{1}, c_{2}\right)$. We first show that for any $0<\varepsilon<1 / 2$,

$$
P\left(\frac{S_{n}}{V_{n, p}} \geq x_{n}\right) \leq \exp \left(-(1-\varepsilon)(p-1) \beta_{p} x_{n}^{p /(p-1)}\right)
$$

provided that $n$ is sufficiently large.

Let $q=p /(p-1), y_{n}$ and $z_{n}$ be defined as in (4.22) and (4.23), respectively, and let $0<\delta<A<\infty$. The values of $\delta$ and $A$ will be specified later on; $\delta$ will be very small, while $A$ will be sufficiently large. Similar to (4.7),

$$
\begin{aligned}
& P\left(\frac{S_{n}}{V_{n, p}} \geq x_{n}\right) \\
& \leq P\left(\frac{S_{n}}{V_{n, p}} \geq x_{n}, \delta x_{n}^{q / p} z_{n}<V_{n, p}<A x_{n}^{q / p} z_{n}\right) \\
& +P\left(\frac{S_{n}}{V_{n, p}} \geq x_{n}, V_{n, p} \geq A x_{n}^{q / p} z_{n}\right)+P\left(V_{n, p} \leq \delta x_{n}^{q / p} z_{n}\right) \\
& \leq P\left(S_{n} \geq \inf _{b=x_{n}^{q / p} / V_{n, p}} \frac{\left(\left(b V_{n, p}\right)^{p} / p+x_{n}^{q} / q\right)}{b}, \delta x_{n}^{q / p} z_{n} \leq V_{n, p} \leq A x_{n}^{q / p} z_{n}\right) \\
& +P\left(\sum_{i=1}^{n} X_{i} I\left\{\left|X_{i}\right| \leq A z_{n}\right\} \geq \frac{A x_{n}^{q} z_{n}}{2}\right) \\
& +P\left(\sum_{i=1}^{n} X_{i} I\left\{\left|X_{i}\right|>A z_{n}\right\} \geq \frac{x_{n} V_{n, p}}{2}\right)+P\left(V_{n, p} \leq \delta x_{n}^{q / p} z_{n}\right) \\
& \leq P\left(p S_{n} \geq \inf _{1 /\left(A z_{n}\right) \leq b \leq 1 /\left(\delta z_{n}\right)} \frac{\left(\left(b V_{n, p}\right)^{p}+(p-1) x_{n}^{q}\right)}{b}\right) \\
& +P\left(\sum_{i=1}^{n} X_{i} I\left\{\left|X_{i}\right| \leq A z_{n}\right\} \geq \frac{A x_{n}^{q} z_{n}}{2}\right) \\
& +P\left(\sum_{i=1}^{n} I\left\{\left|X_{i}\right|>A z_{n}\right\} \geq\left(\frac{x_{n}}{2}\right)^{q}\right)+P\left(V_{n, p} \leq \delta x_{n}^{q / p} z_{n}\right) \\
& :=T_{1}+T_{2}+T_{3}+T_{4} \text {. }
\end{aligned}
$$

From (2.8), (H4), (4.27) and (4.23) it follows that

$$
\begin{aligned}
T_{3} & \leq\left(\frac{2^{q} e n P\left(|X|>A z_{n}\right)}{x_{n}^{q}}\right)^{x_{n}^{q} / 2^{q}} \\
& \leq\left(\frac{4^{q}\left(c_{1}+c_{2}\right) h\left(A z_{n}\right)}{A^{\alpha} z_{n}^{\alpha} y_{n}}\right)^{x_{n}^{q} / 2^{q}} \\
& \leq\left(\frac{5^{q}\left(c_{1}+c_{2}\right) h\left(z_{n}\right)}{A^{\alpha / 2} z_{n}^{\alpha} y_{n}}\right)^{x_{n}^{q} / 2^{q}} \\
& \leq\left(6^{q}\left(c_{1}+c_{2}\right) / A^{\alpha / 2}\right)^{x_{n}^{q} / 2^{q}} \\
& \leq \exp \left(-2(p-1) \beta_{p} x_{n}^{q}\right),
\end{aligned}
$$


provided that $A$ is large enough. Let

$$
t_{p}=\max \left\{2^{p},\left(\frac{600(p-1) \beta_{p}}{c_{1}+c_{2}}\right)^{2 p / \alpha}\right\} \quad \text { and } \quad \delta^{p}=\frac{\left(c_{1}+c_{2}\right) t_{p}^{-1+\alpha /(2 p)}}{300} .
$$

From (4.28) it follows that

$$
\begin{aligned}
T_{4} & \leq \exp \left(t_{p} z_{n}^{-p} \delta^{p} x_{n}^{q} z_{n}^{p}\right) E \exp \left(-t_{p} z_{n}^{-p} V_{n, p}^{p}\right) \\
& =\exp \left(t_{p} \delta^{p} x_{n}^{q}\right)\left(E \exp \left(-t_{p} z_{n}^{-p}|X|^{p}\right)\right)^{n} \\
& \leq \exp \left(t_{p} \delta^{p} x_{n}^{q}-\left(c_{1}+c_{2}\right) n t_{p}^{\alpha /(2 p)} y_{n} / 150\right) \\
& \leq \exp \left(-2(p-1) \beta_{p} x_{n}^{q}\right)
\end{aligned}
$$

by the choice of $t_{p}$ and $\delta^{p}$.

We next estimate $T_{2}$. It is easy to see from Lemma 4.3 that

$$
\begin{aligned}
\sum_{i=1}^{n} \mid & E X_{i} I\left\{\left|X_{i}\right| \leq A z_{n}\right\}|=n| E X I\left\{|X| \leq A z_{n}\right\} \mid \\
& \leq \begin{cases}n E|X| I\left\{|X|>A z_{n}\right\}, & \text { if } 1<\alpha<2, \\
0, & \text { if } \alpha=1 ; \\
n E|X| I\left\{|X| \leq A z_{n}\right\}, & \text { if } 0<\alpha<1,\end{cases} \\
& \leq \begin{cases}2 n \alpha\left(c_{1}+c_{2}\right)\left(A z_{n}\right)^{1-\alpha} h\left(A z_{n}\right) /(\alpha-1), & \text { if } 1<\alpha<2, \\
0, & \text { if } \alpha=1, \\
2 n \alpha\left(c_{1}+c_{2}\right)\left(A z_{n}\right)^{1-\alpha} h\left(A z_{n}\right) /(1-\alpha), & \text { if } 0<\alpha<1 ;\end{cases} \\
& \leq \begin{cases}2 n \alpha\left(c_{1}+c_{2}\right) A^{1-\alpha / 2} z_{n}^{1-\alpha} h\left(z_{n}\right) /(\alpha-1), & \text { if } 1<\alpha<2, \\
0, & \text { if } \alpha=1, \\
2 n \alpha\left(c_{1}+c_{2}\right) A^{1-\alpha / 2} z_{n}^{1-\alpha} h\left(z_{n}\right) /(1-\alpha), & \text { if } 0<\alpha<1 ;\end{cases} \\
\leq A x_{n}^{q} z_{n} / 4 . &
\end{aligned}
$$

Similar to estimating $J_{1}$ in (4.10), by (4.24) (with $p=2$ there), ( $\left.\mathrm{H} 4\right)$ and (4.9) we have

$$
\begin{aligned}
T_{2} \leq & P\left(\sum_{i=1}^{n} X_{i} I\left\{\left|X_{i}\right| \leq A z_{n}\right\}-E X_{i} I\left\{\left|X_{i}\right| \leq A z_{n}\right\} \geq \frac{A x_{n}^{q} z_{n}}{4}\right) \\
\leq & \exp \left(-4(p-1) \beta_{p} x_{n}^{q}\right) \\
\quad & \quad\left(E \exp \left(\frac{16(p-1) \beta_{p}}{A z_{n}}\left(X I\left\{|X| \leq A z_{n}\right\}-E X I\left\{|X| \leq A z_{n}\right\}\right)\right)\right)^{n} \\
\leq & \exp \left(-4(p-1) \beta_{p} x_{n}^{q}\right) \\
\quad & \times\left(1+\left(\frac{16(p-1) \beta_{p}}{A z_{n}}\right)^{2} \exp \left(32(p-1) \beta_{p}\right) E X^{2} I\left\{|X| \leq A z_{n}\right\}\right)^{n}
\end{aligned}
$$




$$
\begin{aligned}
& \leq \exp \left(-4(p-1) \beta_{p} x_{n}^{q}\right) \\
& \times\left(1+\left(\frac{16(p-1) \beta_{p}}{A z_{n}}\right)^{2} \exp \left(32(p-1) \beta_{p}\right)\right. \\
& \left.\times \frac{2 \alpha\left(c_{1}+c_{2}\right)}{2-\alpha}\left(A z_{n}\right)^{2-\alpha} h\left(A z_{n}\right)\right)^{n} \\
& \leq \exp \left(-4(p-1) \beta_{p} x_{n}^{q}\right) \\
& \times\left(1+\left(16(p-1) \beta_{p}\right)^{2} \exp \left(32(p-1) \beta_{p}\right)\right. \\
& \left.\times \frac{2 \alpha\left(c_{1}+c_{2}\right)}{2-\alpha} A^{-\alpha / 2} z_{n}^{-\alpha} h\left(z_{n}\right)\right)^{n} \\
& \leq \exp \left(-4(p-1) \beta_{p} x_{n}^{q}\right)\left(1+2(p-1) \beta_{p} y_{n}\right)^{n} \\
& \leq \exp \left(-2(p-1) \beta_{p} x_{n}^{q}\right),
\end{aligned}
$$

provided that $A$ is chosen sufficiently large.

Finally, we consider $T_{1}$. Let

$$
\theta=(1-\varepsilon / 2)^{-1 / q} \text { and } b_{j}=\theta^{j} /\left(A z_{n}\right), \quad j=0,1,2, \ldots
$$

It follows from Lemma 4.6 that

$$
\begin{aligned}
T_{1}= & P\left(\sup _{1 /\left(A z_{n}\right) \leq b \leq 1 /\left(\delta z_{n}\right)}\left(p b S_{n}-b^{p} V_{n, p}^{p}\right) \geq(p-1) x_{n}^{q}\right) \\
\leq & P\left(\max _{0 \leq j \leq \log _{\theta}(A / \delta)} \sup _{b_{j} \leq b \leq b_{j+1}}\left(p b S_{n}-b^{p} V_{n, p}^{p}\right) \geq(p-1) x_{n}^{q}\right) \\
\leq & P\left(\max _{0 \leq j \leq \log _{\theta}(A / \delta)}\left(p b_{j+1} S_{k}-b_{j}^{p} V_{k}^{p}\right) \geq(p-1) x_{n}^{2}\right) \\
\leq & \sum_{0 \leq j \leq \log _{\theta}(A / \delta)} P\left(p \theta b_{j} S_{n}-b_{j}^{p} V_{n, p}^{p} \geq(p-1) x_{n}^{q}\right) \\
= & \sum_{0 \leq j \leq \log _{\theta}(A / \delta)} P\left(p\left(b_{j} / \theta^{q-1}\right) S_{n}-\left(b_{j} / \theta^{q-1}\right)^{p} V_{n, p}^{2} \geq(p-1)\left(x_{n} / \theta\right)^{q}\right) \\
\leq & \left(1+\log _{\theta}(A / \delta)\right) \sup _{0<b \leq 1 /\left(\delta z_{n}\right)} P\left(p b S_{n}-b^{p} V_{n, p}^{p} \geq(p-1)\left(x_{n} / \theta\right)^{q}\right) \\
\leq & \left(1+\log _{\theta}(A / \delta)\right) \\
& \times \sup _{0<b \leq 1 /\left(\delta z_{n}\right)} \inf \exp \left(-t(p-1)\left(x_{n} / \theta\right)^{q}\right) E \exp \left(t\left(p b S_{n}-b^{p} V_{n, p}^{p}\right)\right) \\
\leq & \left(1+\log _{\theta}(A / \delta)\right) \\
& \times\left(\sup _{0<b \leq 1 /\left(\delta z_{n}\right)} \inf \exp \left(-t(p-1) y_{n} / \theta^{q}\right) E \exp \left(t\left(p b X-|b X|^{p}\right)\right)\right)^{n} \\
\leq & \left(1+\log _{\theta}(A / \delta)\right) \exp \left(-(p-1) \beta_{p} n y_{n} / \theta^{q}+o\left(y_{n}\right) n\right) \\
= & \left(1+\log _{\theta}(A / \delta)\right) \exp \left(-(p-1) \beta_{p} x_{n}^{q} / \theta^{q}+o\left(x_{n}^{q}\right)\right) \\
= & \left(1+\log _{\theta}(A / \delta)\right) \exp \left(-(p-1) \beta_{p}(1-\varepsilon / 2) x_{n}^{q}+o\left(x_{n}^{q}\right)\right) .
\end{aligned}
$$


Putting the above inequalities together yields (4.45) immediately. Based on the same idea as in the proof of (4.2), we next show that

$$
P\left(\frac{S_{n}}{V_{n, p}} \geq x_{n}\right) \geq \exp \left(-(1+\varepsilon)(p-1) \beta_{p} x_{n}^{q}\right) .
$$

Recalling that $\gamma(t)=\alpha t^{\alpha} \Gamma_{p}\left(t, \alpha, c_{1}, c_{2}\right)$, we have $\gamma\left(\beta_{p}\right)=0$. Since $\gamma(t)$ is concave on $(0, \infty)$ and $\lim _{t \downarrow 0} \gamma(t)=0$, it follows from $\gamma\left(\beta_{p}\right)=0$ that $\gamma^{\prime}\left(\beta_{p}\right)<$ 0 . Let $\delta=\varepsilon / 3$ and $\gamma^{\prime}(t)$ be as in (4.30). Put

$$
\begin{gathered}
b:=b_{n, \delta}=\left(-\frac{(1+\delta)(p-1) y_{n}}{\gamma^{\prime}\left(\beta_{p}\right) h\left(z_{n}\right)}\right)^{1 / \alpha} \\
\xi=p b X-|b X|^{p}, \quad \xi_{i}=p b X_{i}-\left|b X_{i}\right|^{p}, \quad i=1,2, \ldots
\end{gathered}
$$

Applying (2.18) again, we have

$$
P\left(\frac{S_{n}}{V_{n, p}} \geq x_{n}\right) \geq P\left(S_{n} \geq \frac{b^{p} V_{n, p}^{p}+(p-1) x_{n}^{q}}{b p}\right)=P\left(\sum_{i=1}^{n} \xi_{i} \geq n(p-1) y_{n}\right) .
$$

Below we verify the condition (4.12). Let $m(\cdot)$ and $\sigma(\cdot)$ be as in Lemma 4.1. From (4.23) it follows that

$$
b \sim \frac{1}{z_{n}}\left(-\frac{(1+\delta)(p-1)}{\gamma^{\prime}\left(\beta_{p}\right)}\right)^{1 / \alpha} .
$$

By Lemma 4.5, ( $\mathrm{H} 2)$ and (4.23), we obtain

$$
\begin{aligned}
E \exp \left(\beta_{p} \xi\right) & =1+o\left(y_{n}\right), \\
E \xi \exp \left(\beta_{p} \xi\right) & =(1+\delta)(p-1) y_{n}+o\left(y_{n}\right), \\
\left(E \xi^{2} \exp \left(\beta_{p} \xi\right)\right)^{1 / 2} / \sqrt{n} & =O\left(\sqrt{y_{n}} / \sqrt{n}\right)=o\left(y_{n}\right)
\end{aligned}
$$

and hence

$$
\begin{gathered}
m\left(\beta_{p}\right)=(1+\delta)(p-1) y_{n}+o\left(y_{n}\right), \\
\sigma\left(\beta_{p}\right) / \sqrt{n}=o\left(y_{n}\right) .
\end{gathered}
$$

Thus, the condition (4.12) is satisfied with $h=\beta_{p}$. Therefore, by Lemma 4.1,

$$
\begin{aligned}
P\left(\sum_{i=1}^{n} \xi_{i} \geq n(p-1) y_{n}\right) & \geq \frac{3}{4}\left(E \exp \left(\beta_{p} \xi\right)\right)^{n} \exp \left(-n \beta_{p} m\left(\beta_{p}\right)-2 \beta_{p} \sigma\left(\beta_{p}\right) \sqrt{n}\right) \\
& \geq \frac{3}{4} \exp \left(o\left(y_{n}\right) n-n(1+\delta)(p-1) \beta_{p} y_{n}\right) \\
& \geq \exp \left(-(1+\varepsilon)(p-1) \beta_{p} x_{n}^{q}\right),
\end{aligned}
$$

as desired.

REMARK 4.3. Similar to Remark 4.1, the convergence in (3.6) is uniform: for arbitrary $0<\varepsilon<1 / 2$, there exist $0<\delta<1, x_{0}>1$ and $n_{0}$ such that for 
any $n \geq n_{0}$ and $x_{0}<x<\delta \sqrt{n}$,

$$
\begin{aligned}
\exp ( & \left.(1+\varepsilon)(p-1) \beta_{p}\left(\alpha, c_{1}, c_{2}\right) x^{p /(p-1)}\right) \\
& \leq P\left(\frac{S_{n}}{V_{n, p}} \geq x\right) \\
& \leq \exp \left(-(1-\varepsilon)(p-1) \beta_{p}\left(\alpha, c_{1}, c_{2}\right) x^{p /(p-1)}\right) .
\end{aligned}
$$

5. Self-normalized law of the iterated logarithm. As we mentioned in Section 1, Griffin and Kuel bs (1989) established an amazing self-normalized law of the iterated logarithm for any i.i.d. random variables in the domain of attraction of a stable law. Equation (1.2) quoted in Section 1 is just a special case of their general result. But, the constant $C$ in (1.2) is unknown. Applying Theorem 3.3, we are able not only to compute the precise constant $C$ but also obtain a law of the iterated logarithm for $S_{n}$ normalized by $V_{n, p}$.

THEOREM 5.1. Under the conditions of Theorem 3.3, we have

$$
\limsup _{n \rightarrow \infty} \frac{S_{n}}{(\log \log n)^{(p-1) / p} V_{n, p}}=\left((p-1) \beta_{p}\left(\alpha, c_{1}, c_{2}\right)\right)^{(1-p) / p} \quad \text { a.s. }
$$

In particular, if $X$ is symmetric, then

$$
\limsup _{n \rightarrow \infty} \frac{S_{n}}{(\log \log n)^{(p-1) / p} V_{n, p}}=\left((p-1) \beta_{p}(\alpha)\right)^{(1-p) / p} \quad \text { a.S., }
$$

where $\beta_{p}\left(\alpha, c_{1}, c_{2}\right)$ and $\beta(\alpha)$ are defined as in Theorem 3.3.

To prove the upper bound of the limsup, we need a strong version of (4.45).

Proposition 5.1. Under the conditions of Theorem 3.3, for any $0<\varepsilon<1 / 2$ there exists $\theta>1$ such that

$$
P\left(\max _{n \leq k \leq \theta n} \frac{S_{k}}{V_{k, p}} \geq x_{n}\right) \leq \exp \left(-(1-\varepsilon)(p-1) \beta_{p}\left(\alpha, c_{1}, c_{2}\right) x_{n}^{p /(p-1)}\right)
$$

for every $n$ sufficiently large.

Proof. Let $q=p /(p-1)$ and $\eta=\left(1-(1-\varepsilon / 2)^{1 /(2 q)}\right) / 3$. Clearly,

$$
\begin{aligned}
P\left(\max _{n \leq k \leq \theta n} \frac{S_{k}}{V_{k, p}} \geq x_{n}\right) \leq & P\left(\frac{S_{n}}{V_{n, p}} \geq(1-3 \eta) x_{n}\right) \\
& +P\left(\max _{n<k \leq \theta n} \frac{S_{k}-S_{n}}{V_{k, p}} \geq 3 \eta x_{n}\right) .
\end{aligned}
$$

By Theorem 3.3, we have

$$
P\left(\frac{S_{n}}{V_{n, p}} \geq(1-3 \eta) x_{n}\right) \leq \exp \left(-(1-\varepsilon / 2)(p-1) \beta_{p}\left(\alpha, c_{1}, c_{2}\right) x_{n}^{q}\right),
$$

provided that $n$ is sufficiently large. 
Below we estimate the second term on the right-hand side of (5.4). Let $z_{n}$ be as in (4.23) and let $\delta>0$. Write

$$
\begin{aligned}
& P\left(\max _{n<k \leq \theta n} \frac{S_{k}-S_{n}}{V_{k, p}} \geq 3 \eta x_{n}\right) \\
& \leq P\left(\max _{n<k \leq \theta n} \frac{\sum_{i=n+1}^{k} X_{i} I\left\{\left|X_{i}\right| \leq z_{n}\right\}}{V_{k, p}} \geq 2 \eta x_{n}\right) \\
&+P\left(\max _{n<k \leq \theta n} \frac{\sum_{i=n+1}^{k}\left|X_{i}\right| I\left\{\left|X_{i}\right| \geq z_{n}\right\}}{V_{k, p}} \geq \eta x_{n}\right) \\
& \leq P\left(\max _{n<k \leq \theta n} \sum_{i=n+1}^{k} X_{i} I\left\{\left|X_{i}\right| \leq z_{n}\right\} \geq 2 \eta \delta x_{n}^{q} z_{n}\right) \\
&+P\left(V_{n, p} \leq \delta x_{n}^{q / p} z_{n}\right)+P\left(\sum_{i=n+1}^{[\theta n]} I\left\{\left|X_{i}\right| \geq z_{n}\right\} \geq\left(\eta x_{n}\right)^{q}\right) .
\end{aligned}
$$

By (4.48), there is $\delta>0$ such that

$$
P\left(V_{n, p} \leq \delta x_{n}^{q / p} z_{n}\right) \leq \exp \left(-2(p-1) \beta_{p}\left(\alpha, c_{1}, c_{2}\right) x_{n}^{q}\right) .
$$

Similar to (4.47), we have

$$
\begin{aligned}
P\left(\sum_{i=n+1}^{[\theta n]} I\left\{\left|X_{i}\right| \geq z_{n}\right\} \geq\left(\eta x_{n}\right)^{q}\right) & \leq\left(\frac{(\theta-1) n P\left(|X| \geq z_{n}\right)}{\left(\eta x_{n}\right)^{q}}\right)^{\left(\eta x_{n}\right)^{q}} \\
& \leq\left(\frac{2 e\left(c_{1}+c_{2}\right)(\theta-1) n h\left(z_{n}\right)}{\left(\eta x_{n}\right)^{q} z_{n}^{\alpha}}\right)^{\left(\eta x_{n}\right)^{q}} \\
& \leq\left(\frac{6(\theta-1)\left(c_{1}+c_{2}\right)}{\eta^{q}}\right)^{\eta^{q} x_{n}^{q}} \\
& \leq \exp \left(-2(p-1) \beta_{p}\left(\alpha, c_{1}, c_{2}\right) x_{n}^{q}\right),
\end{aligned}
$$

as long as $\theta$ is very close to one. In view of the proof of (4.49), if $\theta-1>0$ is chosen to be sufficiently small,

$$
\sum_{i=n+1}^{[\theta n]}\left|E X_{i} I\left\{\left|X_{i}\right| \leq z_{n}\right\}\right| \leq K(\theta-1) x_{n}^{q} z_{n} \leq \frac{1}{2} \eta \delta x_{n}^{q} z_{n}
$$

and

$$
\begin{aligned}
\sum_{i=n+1}^{[\theta n]} \operatorname{Var} X_{i} I\left\{\left|X_{i}\right| \leq z_{n}\right\} & \leq(\theta-1) n E X^{2} I\left\{|X| \leq z_{n}\right\} \\
& \leq \frac{2(\theta-1) n \alpha\left(c_{1}+c_{2}\right)}{2-\alpha} z_{n}^{2-\alpha} h\left(z_{n}\right) \\
& \leq K(\theta-1) x_{n}^{q} z_{n}^{2} \leq \frac{1}{8} \eta \delta x_{n}^{q} z_{n}^{2}
\end{aligned}
$$


where $K$ is a constant depending only on $\alpha, c_{1}$ and $c_{2}$. Therefore, by the Ottaviani maximum inequality and (4.9),

$$
\begin{aligned}
& P\left(\max _{n<k \leq \theta n} \sum_{i=n+1}^{k} X_{i} I\left\{\left|X_{i}\right| \leq z_{n}\right\} \geq 2 \eta \delta x_{n}^{q} z_{n}\right) \\
& \leq 2 P\left(\sum_{i=n+1}^{[\theta n]} X_{i} I\left\{\left|X_{i}\right| \leq z_{n}\right\}-E X_{i} I\left\{\left|X_{i}\right| \leq z_{n}\right\} \geq \eta \delta x_{n}^{q} z_{n}\right) \\
& \leq 2 \exp \left(-4(p-1) \beta_{p}\left(\alpha, c_{1}, c_{2}\right) x_{n}^{q}\right) \\
& \times\left(E \operatorname { e x p } \left(\frac{4(p-1) \beta_{p}\left(\alpha, c_{1}, c_{2}\right)}{\eta \delta z_{n}}\right.\right. \\
&\left.\left.\quad \times\left(X I\left\{|X| \leq z_{n}\right\}-E X I\left\{|X| \leq z_{n}\right\}\right)\right)\right)^{[\theta n]-n} \\
& \leq 2 \exp \left(-4(p-1) \beta_{p}\left(\alpha, c_{1}, c_{2}\right) x_{n}^{q}\right)\left(1+K z_{n}^{-2} E X^{2} I\left\{|X| \leq z_{n}\right\}\right)^{(\theta-1) n} \\
& \leq 2 \exp \left(-4(p-1) \beta_{p}\left(\alpha, c_{1}, c_{2}\right) x_{n}^{q}\right)\left(1+K z_{n}^{-\alpha} h\left(z_{n}\right)\right)^{(\theta-1) n} \\
& \leq 2 \exp \left(-4(p-1) \beta_{p}\left(\alpha, c_{1}, c_{2}\right) x_{n}^{q}\right)\left(1+\frac{K x_{n}^{q}}{n}\right)^{(\theta-1) n} \\
& \leq 2 \exp \left(-4(p-1) \beta_{p}\left(\alpha, c_{1}, c_{2}\right) x_{n}^{q}+K(\theta-1) x_{n}^{q}\right) \\
& \leq 2 \exp \left(-2(p-1) \beta_{p}\left(\alpha, c_{1}, c_{2}\right) x_{n}^{q}\right),
\end{aligned}
$$

where $K$ stands for a constant depending only on $\alpha, p, c_{1}, c_{2}, \eta$ and $\delta$. Putting together the above inequalities yields

$$
P\left(\max _{n<k \leq \theta n} \frac{S_{k}-S_{n}}{V_{k, p}} \geq 3 \eta x_{n}\right) \leq 4 \exp \left(-2(p-1) \beta_{p}\left(\alpha, c_{1}, c_{2}\right) x_{n}^{q}\right) .
$$

This proves (5.3), by (5.5), (5.4) and (5.6).

Proof of Theorem 5.1. By the subsequence method, it follows from Proposition 5.1 that

$$
\limsup _{n \rightarrow \infty} \frac{S_{n}}{(\log \log n)^{(p-1) / p} V_{n, p}} \leq\left((p-1) \beta_{p}\left(\alpha, c_{1}, c_{2}\right)\right)^{(1-p) / p} \quad \text { a.s. }
$$

To prove the lower bound of the limsup, let $q=p /(p-1), \tau>1$ and $n_{k}=\left[e^{k^{\tau}}\right], k=1,2, \ldots$ Note that

$$
\begin{aligned}
& \limsup _{n \rightarrow \infty} \frac{S_{n}}{(\log \log n)^{1 / q} V_{n, p}} \\
& \quad \geq \limsup _{k \rightarrow \infty} \frac{S_{n_{k}}}{\left(\log \log n_{k}\right)^{1 / q} V_{n_{k}, p}} \\
& \quad \geq \limsup _{k \rightarrow \infty} \frac{S_{n_{k}}-S_{n_{k-1}}}{\left(\log \log n_{k}\right)^{1 / q} V_{n_{k}, p}}+\liminf _{k \rightarrow \infty} \frac{S_{n_{k-1}}}{\left(\log \log n_{k}\right)^{1 / q} V_{n_{k}, p}}
\end{aligned}
$$




$$
\begin{aligned}
= & \limsup _{k \rightarrow \infty} \frac{\left(V_{n_{k}, p}^{p}-V_{n_{k-1}, p}^{p}\right)^{1 / p}}{V_{n_{k}, p}} \frac{S_{n_{k}}-S_{n_{k-1}}}{\left(\log \log n_{k}\right)^{1 / q}\left(V_{n_{k}, p}^{p}-V_{n_{k-1}, p}^{p}\right)^{1 / p}} \\
& +\liminf _{k \rightarrow \infty} \frac{V_{n_{k-1}, p}}{V_{n_{k}, p}} \frac{S_{n_{k-1}}}{\left(\log \log n_{k}\right)^{1 / q} V_{n_{k-1}, p}} .
\end{aligned}
$$

Since $\left.\left\{\left(S_{n_{k}}-S_{n_{k-1}}\right) /\left(V_{n_{k}, p}^{p}-V_{n_{k-1}, p}^{p}\right)^{1 / p}\right), k \geq 1\right\}$ are independent, it follows from Theorem 3.3 and the Borel-Cantelli lemma that

$$
\begin{gathered}
\limsup _{k \rightarrow \infty} \frac{S_{n_{k}}-S_{n_{k-1}}}{\left(\log \log n_{k}\right)^{1 / q}\left(V_{n_{k}, p}^{p}-V_{n_{k-1}, p}^{p}\right)^{1 / p}} \\
\geq \frac{1}{\tau^{2}\left((p-1) \beta_{p}\left(\alpha, c_{1}, c_{2}\right)\right)^{1 / q}} \text { a.s. }
\end{gathered}
$$

On the other hand, by Proposition 5.2 of Griffin and Kuelbs (1989),

$$
\lim _{k \rightarrow \infty} \frac{V_{n_{k}, p}}{V_{n_{k-1}, p}}=\infty \quad \text { a.s. }
$$

Hence, by (5.8), (5.9), (5.10) and (5.7),

$$
\limsup _{n \rightarrow \infty} \frac{S_{n}}{(\log \log n)^{1 / q} V_{n, p}} \geq \frac{1}{\tau^{2}\left((p-1) \beta_{p}\left(\alpha, c_{1}, c_{2}\right)\right)^{1 / q}} \text { a.s. }
$$

This proves (5.1), by (5.7), (5.11) and the arbitrariness of $\tau>1$.

6. Limit distribution of self-normalized sums. Self-normalized sums have been studied previously in connection with weak convergence [see Darling (1952), Logan, Mallows, Rice and Shepp (1973), Csörgő and Horváth (1988), and Hahn, Kuel bs and Weiner (1990)]. Logan, Mallows, Rice and Shepp (1973) proved that all limit laws of $S_{n} / V_{n}$ for $X$ in the domain of attraction of a stable law have a sub-Gaussian tail which depends in a complicated way on the parameter $\alpha$.

TheoREm 6.1* (Logan, Mallows, Rice and Shepp). Under the condition of Theorem 3.3, the limiting density function $p(x)$ of $S_{n} / V_{n}$ exists and satisfies as $x \rightarrow \infty$,

$$
p(x) \sim \frac{1}{\alpha}\left(\frac{2}{\pi}\right)^{1 / 2} \tau_{\alpha} \exp \left(-\frac{1}{2} x^{2} \tau_{\alpha}^{2}\right)
$$

for some $\tau_{\alpha}>0$.

On the basis of both mathematical simplicity and numerical evidence, they conjectured [cf. pages 799-800 in Logan, Mallows, Rice and Shepp (1973)] that $\tau_{\alpha}$ is the solution of

$$
\begin{cases}c_{1} D_{\alpha}(-\tau)+c_{2} D_{\alpha}(\tau)=0, & \text { if } \alpha \neq 1, \\ \frac{\exp \left(\tau^{2} / 2\right)}{\tau}-\int_{0}^{\tau} \exp \left(x^{2} / 2\right) d x=0, & \text { if } \alpha=1,\end{cases}
$$


where $D_{\alpha}(x)$ is the parabolic cylinder function [cf. pages 795 and 807 in Logan, Mallows, Rice and Shepp (1973)].

Applying Theorem 3.2, we can determine that the above conjecture is true.

TheOREM 6.1. Let $\beta\left(\alpha, c_{1}, c_{2}\right)$ beas in Theorem 3.2 and let $\tau_{\alpha}$ bethesolution of (6.2). Then $\tau_{\alpha}=\sqrt{2 \beta\left(\alpha, c_{1}, c_{2}\right)}$ and (6.1) holds.

Proof. By Theorem 3.2 and Theorem $6.1^{*}$, it suffices to show that $\sqrt{2 \beta\left(\alpha, c_{1}, c_{2}\right)}$ is the solution of (6.2). Recalling the following properties of $D_{\nu}(z)$ :

$$
\begin{gathered}
D_{\nu}(z)=\frac{\exp \left(-z^{2} / 4\right)}{\Gamma(-\nu)} \int_{0}^{\infty} \exp \left(-z x-x^{2} / 2 x^{-\nu-1}\right) d x \text { for } \nu<0 \\
D_{v+1}(z)-z D_{\nu}(z)+\nu D_{\nu-1}(z)=0 \text { for } v \in R^{1}
\end{gathered}
$$

we have

$$
D_{\alpha}(z)= \begin{cases}\frac{\alpha e^{-z^{2} / 2}}{\Gamma(1-\alpha)} \int_{0}^{\infty} \frac{1-\exp \left(-z x-x^{2} / 2\right)}{x^{\alpha+1}} d x, & \text { if } 0<\alpha<1 \\ \frac{\alpha(1-\alpha) \exp \left(-z^{2} / 2\right)}{\Gamma(2-\alpha)} \int_{0}^{\infty} \frac{1-z x-\exp \left(-z x-x^{2} / 2\right)}{x^{\alpha+1}} d x & \text { if } 1<\alpha<2\end{cases}
$$

Let $\Gamma\left(\beta, \alpha, c_{1}, c_{2}\right)$ be defined as in (3.4). It is easy to see that

$$
\begin{aligned}
& c_{1} D_{\alpha}(-\sqrt{2 \beta})+c_{2} D_{\alpha}(\sqrt{2 \beta}) \\
& = \begin{cases}\frac{\alpha e^{-\beta}}{\Gamma(1-\alpha)}\left(\frac{\beta}{2}\right)^{\alpha / 2} \Gamma\left(\beta, \alpha, c_{1}, c_{2}\right), & \text { if } 0<\alpha<1, \\
\frac{\alpha(1-\alpha) e^{-\beta}}{\Gamma(2-\alpha)}\left(\frac{\beta}{2}\right)^{\alpha / 2} \Gamma\left(\beta, \alpha, c_{1}, c_{2}\right), & \text { if } 1<\alpha<2 .\end{cases}
\end{aligned}
$$

This proves that $\sqrt{2 \beta\left(\alpha, c_{1}, c_{2}\right)}$ is the solution of (6.2) for $\alpha \neq 1$.

We next deal with the case of $\alpha=1$. Write $\beta=\tau^{2} / 2$. Since

$$
\begin{aligned}
\int_{0}^{\infty} & \frac{2-\exp \left(2 x-x^{2} / \beta\right)-\exp \left(-2 x-x^{2} / \beta\right)}{x^{2}} d x \\
& =\frac{\tau}{2} \int_{0}^{\infty} \frac{2-\exp \left(x \tau-x^{2} / 2\right)-\exp \left(-x \tau-x^{2} / 2\right)}{x^{2}} d x,
\end{aligned}
$$

it suffices to verify that

$$
\begin{gathered}
\int_{0}^{\infty} \frac{2-\exp \left(x \tau-x^{2} / 2\right)-\exp \left(-x \tau-x^{2} / 2\right)}{x^{2}} d x \\
=\sqrt{2 \pi}\left(\exp \left(\tau^{2} / 2\right)-\tau \int_{0}^{\tau} \exp \left(x^{2} / 2\right) d x\right) .
\end{gathered}
$$


One has

$$
\begin{aligned}
\int_{0}^{\infty} \frac{2-}{\exp \left(x \tau-x^{2} / 2\right)-\exp \left(-x \tau-x^{2} / 2\right)} & x^{2} d x \\
= & \int_{0}^{\infty} \frac{(x-\tau) \exp \left(x \tau-x^{2} / 2\right)+(x+\tau) \exp \left(-x \tau-x^{2} / 2\right)}{x} d x \\
= & \int_{0}^{\infty}\left(\exp \left(x \tau-x^{2} / 2\right)+\exp \left(-x \tau-x^{2} / 2\right)\right) d x \\
& -\tau \int_{0}^{\infty} \frac{\exp \left(-x^{2} / 2\right)(\exp (x \tau)-\exp (-x \tau))}{x} d x \\
= & \exp \left(\tau^{2} / 2\right)\left(\int_{0}^{\infty} \exp \left(-(x-\tau)^{2} / 2\right) d x+\int_{0}^{\infty} \exp \left(-(x+\tau)^{2} / 2\right) d x\right) \\
& -2 \tau \int_{0}^{\infty} \exp \left(-x^{2} / 2\right) \sum_{i=0}^{\infty} \frac{x^{2 i} \tau^{2 i+1}}{(2 i+1) !} d x \\
= & \exp \left(\tau^{2} / 2\right)\left(\int_{0}^{\tau} \exp \left(-x^{2} / 2\right) d x+\int_{0}^{\infty} \exp \left(-x^{2} / 2\right) d x\right. \\
& -\tau \sqrt{2 \pi} \sum_{i=0}^{\infty} \frac{(2 i) ! \tau^{2 i+1}}{(2 i+1) ! i ! 2^{i}} \\
= & \sqrt{2 \pi}\left(\exp \left(\tau^{2} / 2\right)-\tau \sum_{i=0}^{\infty} \int_{0}^{\tau} \frac{x^{2 i}}{i ! 2^{i}} d x\right) \\
= & \sqrt{2 \pi}\left(\exp \left(\tau^{2} / 2\right)-\tau \int_{0}^{\tau} \exp \left(x^{2} / 2\right) d x\right),
\end{aligned}
$$

as desired. This completes the proof of Theorem 6.1.

7. Asymptotic probability of the t-statistic. Consider Student's $t$ statistic $T_{n}$ defined by

$$
T_{n}=\frac{\sum_{i=1}^{n} X_{i}}{\sqrt{n}} /\left(\frac{1}{n-1} \sum_{j=1}^{n}\left(X_{j}-\bar{X}\right)^{2}\right)^{1 / 2} .
$$

Clearly, $T_{n}$ and $S_{n} / V_{n}$ are closely related via the following identity:

$$
T_{n}=\frac{S_{n}}{V_{n}}\left(\frac{n-1}{n-\left(S_{n} / V_{n}\right)^{2}}\right)^{1 / 2} .
$$

Since $x /\left(n-x^{2}\right)^{1 / 2}$ is increasing on $(-\sqrt{n}, \sqrt{n})$, it follows from (7.1) that

$$
\left\{T_{n} \geq t\right\}=\left\{\frac{S_{n}}{V_{n}} \geq t\left(\frac{n}{n+t^{2}-1}\right)^{1 / 2}\right\} .
$$


The above fact was pointed out by Efron (1969), who studied the limiting distribution of $S_{n} / V_{n}$ for $X$ in the domain of a stable law. Hotelling (1961) also studied the asymptotics of $T_{n}$ for long-tailed $X$ and has additional references.

With the help of (7.2), the following large deviation type results as well as the laws of the iterated logarithm for $t$-statistic are immediate consequences of Theorems 1.1, 3.1, 3.2 and 5.1.

Theorem 7.1. (a) Assume that either $E X \geq 0$ or $E X^{2}=\infty$. Then

$$
\lim _{n \rightarrow \infty} P\left(T_{n} \geq x \sqrt{n}\right)^{1 / n}=\operatorname{supinf}_{c \geq 0} E \exp \left(t\left(c X-\frac{x\left(X^{2}+c^{2}\right)}{2 \sqrt{1+x^{2}}}\right)\right)
$$

for $x>E X /(\operatorname{Var} X)^{1 / 2}$.

(b) Under the conditions of Theorem 3.1, we have

$$
\lim _{n \rightarrow \infty} x_{n}^{-2} \ln P\left(T_{n} \geq x_{n}\right)=-1 / 2
$$

for every sequence $\left\{x_{n}, n \geq 1\right\}$ of positive numbers with $x_{n} \rightarrow \infty$ and $x_{n}=$ $o(\sqrt{n})$ as $n \rightarrow \infty$, and

$$
\limsup _{n \rightarrow \infty} \frac{T_{n}}{(2 \log \log n)^{1 / 2}}=1 \text { a.s. }
$$

(c) Under the conditions of Theorem 3.2, we have

$$
\lim _{n \rightarrow \infty} x_{n}^{-2} \ln P\left(T_{n} \geq x_{n}\right)=-\beta\left(\alpha, c_{1}, c_{2}\right)
$$

for every sequence $\left\{x_{n}, n \geq 1\right\}$ of positive numbers with $x_{n} \rightarrow \infty$ and $x_{n}=$ $o(\sqrt{n})$ as $n \rightarrow \infty$, and

$$
\limsup _{n \rightarrow \infty} \frac{T_{n}}{(\log \log n)^{1 / 2}}=1 / \sqrt{\beta\left(\alpha, c_{1}, c_{2}\right)} \text { a.s. }
$$

8. Erdös-Rényi-Shepp law of Iarge numbers. Let $c>0$. We are concerned with the limiting behavior of

$$
U_{n}=\max _{0 \leq i \leq n}\left(S_{i+[c \log n]}-S_{i}\right) .
$$

The classical Erdős-Rényi-Shepp law of large numbers [see Erdős and Rényi (1970) and Shepp (1966)] says that if $E X=0, E e^{t_{0} X}<\infty$ for some $t_{0}>0$, then

$$
\lim _{n \rightarrow \infty} \frac{U_{n}}{[c \log n]}=\lambda(c) \quad \text { a.s., }
$$

where $\lambda(c)=\sup \left\{x\right.$ : $\left.\inf _{t \geq 0} \exp (-t x) E \exp (t X) \geq \exp (-1 / c)\right\}$. The result was refined by S. Csörgö (1979) and M. Csörgö and Steinebach (1981), while the exact rate of convergence of $U_{n} /[c \log n]$ was determined by Deheuvels, Devroye and Lynch (1986). We remark that the condition $E \exp \left(t_{0} X\right)<\infty$ is essential for an (8.1) type result. Motivated by the self-normalized law of the iterated logarithm of Griffin and Kuel bs (1989), Csörgő and Shao (1994) were 
the first to consider a self-normalized Erdös-Rényi-Shepp type law of Iarge numbers and obtain the following result.

Assuming $E X \geq 0$, we have

$$
\lim _{n \rightarrow \infty} \max _{0 \leq k \leq n} \frac{S_{k+[c \log n]}-S_{k}}{\sum_{i=k+1}^{k+[c \log n]}\left(X_{i}^{2}+1\right)}=\Lambda(c) \quad \text { a.S. }
$$

where $\Lambda(c)=\sup \left\{x \geq 0: \inf _{t \geq 0} E \exp \left(t\left(X-x\left(X^{2}+1\right)\right)\right) \geq \exp (-1 / c)\right\}$.

Applying Theorem 1.1, we are able to establish another self-normalized Erdős-Rényi-Shepp type law of large numbers, which may be of more interest from a statistical point of view.

TheOREM 8.1. Assume that either $E X \geq 0$ or $E X^{2}=\infty$. Then

$$
\lim _{n \rightarrow \infty} \max _{0 \leq k \leq n} \frac{S_{k+[c \log n]}-S_{k}}{\sqrt{[c \log n] \sum_{i=k+1}^{k+[c \log n]} X_{i}^{2}}}=\kappa(c) \text { a.s. }
$$

for any $c>1 / \ln (1 / P(X=0))$, where $\kappa(c)=\inf \{x \geq 0: f(x)<\exp (-1 / c)\}$ and $f(x)=\sup _{b \geq 0}$ inf $_{t \geq 0} E \exp \left(t\left(b X-x\left(X^{2}+b^{2}\right) / 2\right)\right)$.

Let $x_{0}=E X / \sqrt{E X^{2}}$. If one could prove that $f(x)$ is continuous and strictly monotone decreasing for $x_{0} \leq x \leq 1$, then the proof of (8.2) would be quite standard [cf., e.g., S. Csörgő (1979) or Csörgő and Révész (1981)]. However, we are unable to verify the strict monotonicity, so we have to use the next lemma instead. Its proof is given in the Appendix.

LEMma 8.1. Let $0<\delta<1 / 2$. Define

$$
f_{\delta}(x)=\operatorname{supinf}_{b \geq 0} \exp \left((t \delta)^{2} / 2\right) E \exp \left(t\left(b X-x\left(X^{2}+b^{2}\right) / 2\right)\right) \text {. }
$$

Then,

$$
f\left(x_{0}\right)=f_{\delta}\left(x_{0}\right)=1
$$

$$
f(1)=\sup _{b \geq 0} P(X=b) \quad \text { and } \quad f(x)=P(X=0) \quad \text { for } x>1 .
$$

Also, $f_{\delta}(x)$ is continuous and strictly monotone decreasing for $x \geq x_{0}$. More over, $f(x)$ is continuous for $x>x_{0}$.

Proof of Theorem 8.1. Let $0<\delta<1 / 2,\left\{Y, Y_{n}, n \geq 1\right\}$ be i.i.d. standard normal random variables independent of $\left\{X_{n}, n \geq 1\right\}$. Define

$$
\kappa_{\delta}(c)=\inf \left\{x \geq 0: f_{\delta}(x)<\exp (-1 / c)\right\} .
$$

From the proof of Theorem 1.1 we obtain that

$$
\lim _{m \rightarrow \infty} P\left(\frac{\sum_{i=1}^{m}\left(X_{i}+\delta Y_{i}\right)}{\sqrt{m \sum_{i=1}^{m} X_{i}^{2}}} \geq x\right)^{1 / m}=f_{\delta}(x)
$$


for every $x>E X / \sqrt{E X^{2}}$. Therefore, by Lemma 8.1 and a general version of Erdős-Rényi-Shepp laws due to S. Csörgö (1979) [cf. Steinebach (1980)]

$$
\lim _{n \rightarrow \infty} \max _{0 \leq k \leq n} \frac{\sum_{i=k+1}^{k+[c \log n]}\left(X_{i}+\delta Y_{i}\right)}{\sqrt{[c \log n] \sum_{i=k+1}^{k+[c \log n]} X_{i}^{2}}}=\kappa_{\delta}(c) \text { a.s. }
$$

for every $c>1 / \ln (1 / P(X=0))$. To finish the proof of $(8.2)$, we only need to prove that

$$
\lim _{\delta \downarrow 0} \kappa_{\delta}(c)=\kappa(c)
$$

and

$$
\limsup _{n \rightarrow \infty} \max _{0 \leq k \leq n} \frac{\left|\sum_{i=k+1}^{k+[c \log n]} Y_{i}\right|}{\sqrt{[c \log n] \sum_{i=k+1}^{k+[c \log n]} X_{i}^{2}}} \leq D_{0} \quad \text { a.s. }
$$

for some finite constant $D_{0}$. Clearly,

$$
\liminf _{\delta \downarrow 0} \kappa_{\delta}(c) \geq \kappa(c)
$$

for $f_{\delta}(x) \geq f(x)$ for any $\delta>0$. On the other hand, by the definition of $\kappa(c)$

$$
\forall \varepsilon>0, \quad \exists 0<\eta<\varepsilon, \quad f(\kappa(c)+\eta)<\exp (-1 / c) .
$$

From Lemma 2.1 we find that

$$
\lim _{\delta \downarrow 0} f_{\delta}(\kappa(c)+\eta)=f(\kappa(c)+\eta)<\exp (-1 / c) .
$$

Therefore, there exists $\delta_{\varepsilon}>0$ such that

$$
f_{\delta}(\kappa(c)+\eta)<\exp (-1 / c) \text { for every } 0<\delta<\delta_{\varepsilon} .
$$

Thus, in terms of the definition of $\kappa_{\delta}(c)$,

$$
\forall 0<\delta<\delta_{\varepsilon}, \quad \kappa_{\delta}(c) \leq \kappa(c)+\eta<\kappa(c)+\varepsilon,
$$

which together with (8.9) implies (8.7).

As to (8.8), letting $p_{m}=e^{m / c}$, we have

$$
\begin{aligned}
& \underset{n \rightarrow \infty}{\limsup } \max _{0 \leq k \leq n} \frac{\left|\sum_{i=k+1}^{k+[c \log n]} Y_{i}\right|}{\sqrt{[c \log n] \sum_{i=k+1}^{k+[c \log n]} X_{i}^{2}}} \\
& \quad \leq \limsup _{m \rightarrow \infty} \max _{p_{m} \leq n<p_{m+1}} \max _{0 \leq k \leq n} \frac{\left|\sum_{i=k+1}^{k+[c \log n]} Y_{i}\right|}{\sqrt{[c \log n] \sum_{i=k+1}^{k+[c \log n]} X_{i}^{2}}} \\
& \quad=\limsup _{m \rightarrow \infty} \max _{p_{m} \leq n<p_{m+1}} \max _{0 \leq k \leq n} \frac{\left|\sum_{i=k+1}^{k+m} Y_{i}\right|}{\sqrt{m \sum_{i=k+1}^{k+m} X_{i}^{2}}} \\
& \quad \leq \limsup _{m \rightarrow \infty} \max _{0 \leq k \leq \exp ((m+1) / c)} \frac{\left|\sum_{i=k+1}^{k+m} Y_{i}\right|}{\sqrt{m \sum_{i=k+1}^{k+m} X_{i}^{2}}} .
\end{aligned}
$$


Since $\lim _{d \downarrow 0}$ inf $_{t \geq 0} E \exp \left(t\left(d^{2}-X^{2}\right)\right)=P(X=0)$ and $P(X=0)<\exp (-1 / c)$, choose $d_{0}>0$ such that

$$
\inf _{t \geq 0} E \exp \left(t\left(d_{0}^{2}-X^{2}\right)\right)<\exp (-1 / c)
$$

Put $D_{0}=2 /\left(d_{0} \sqrt{c}\right)$. Observe that

$$
\begin{aligned}
& P\left(\max _{0 \leq k \leq \exp ((m+1) / c)} \frac{\left|\sum_{i=k+1}^{k+m} Y_{i}\right|}{\sqrt{m \sum_{i=k+1}^{k+m} X_{i}^{2}}} \geq D_{0}\right) \\
& \quad \leq \exp ((m+1) / c) P\left(\frac{\left|\sum_{i=1}^{m} Y_{i}\right|}{\sqrt{m \sum_{i=1}^{m} X_{i}^{2}}} \geq D_{0}\right) \\
& \quad \leq \exp ((m+1) / c)\left(P\left(\left|\sum_{i=1}^{m} Y_{i}\right| \geq 2 m / \sqrt{c}\right)+P\left(\sum_{i=1}^{m} X_{i}^{2} \leq m d_{0}^{2}\right)\right) \\
& \quad \leq \exp ((m+1) / c)\left(2 \exp \left(-\frac{2 m}{c}\right)+\left(\inf _{t \geq 0} E \exp \left(t\left(d_{0}^{2}-X^{2}\right)\right)\right)^{m}\right),
\end{aligned}
$$

which is summable over $m$, by (8.10). This proves (8.8).

The proof of Theorem 8.1 is now complete.

Applying Theorem 1.2 instead of Theorem 1.1, along the same line of the proof of Theorem 8.1, one can obtain a more general result.

Theorem 8.2. Let $p>1$. Assume that either $E X \geq 0$ or $E|X|^{p}=\infty$. Then

$$
\lim _{n \rightarrow \infty} \max _{0 \leq k \leq n} \frac{S_{k+[c \log n]}-S_{k}}{[c \log n]^{1-1 / p}\left(\sum_{i=k+1}^{k+[c \log n]}\left|X_{i}\right|^{p}\right)^{1 / p}}=\kappa(p, c) \text { a.s. }
$$

for any $c>1 / \ln (1 / P(X=0))$, where

$$
\begin{array}{r}
\kappa(p, c)=\inf \left\{x \geq 0: \operatorname{supinf}_{b \geq 0} E \exp \left(t\left(b X-x\left(\frac{1}{p}|X|^{p}+\frac{p-1}{p} b^{p /(p-1)}\right)\right)\right)\right. \\
<\exp (-1 / c)\} .
\end{array}
$$

\section{APPENDIX}

Proof of Lemma 2.1. We first show that

$$
\operatorname{limsupinf}_{k \rightarrow \infty} \exp \left(t^{2} / 2\right) E \exp \left(t\left(b X-x\left(X^{2}+b^{2}\right) / 2\right)\right)=0
$$

uniformly in $x \in[a, 1]$.

Let $\left\{m_{k}, k \geq 1\right\}$ be a sequence of positive numbers such that as $k \rightarrow \infty$,

(A.2) $\quad m_{k} \rightarrow \infty, \quad \exp \left(m_{k} / a\right) P(|X|>\sqrt{k}) \rightarrow 0, \quad m_{k}=o(\sqrt{k})$. 
Notice that by (A.2)

$$
\begin{aligned}
& \lim _{k \rightarrow \infty} \sup _{b \geq k} \inf _{t \geq 0} \exp \left(t^{2} / 2\right) E \exp \left(t\left(b X-x\left(X^{2}+b^{2}\right) / 2\right)\right) \\
& \leq \limsup _{k \rightarrow \infty} \sup _{b \geq k} \exp \left(\left(m_{k} / b^{2}\right)^{2} / 2\right) E \exp \left(\left(m_{k} / b^{2}\right)\left(b X-a\left(X^{2}+b^{2}\right) / 2\right)\right) \\
& \leq \limsup _{k \rightarrow \infty} \sup _{b \geq k} \exp \left(\left(m_{k} / b^{2}\right)^{2} / 2\right)\left(E \exp \left(\left(m_{k} / b^{2}\right)\left(b X-a b^{2} / 2\right)\right) I\{|X| \leq \sqrt{k}\}\right. \\
& \left.+E \exp \left(\left(m_{k} / b^{2}\right)\left(-a(X-b / a)^{2} / 2+b^{2} /(2 a)\right)\right) I\{|X|>\sqrt{k}\}\right) \\
& \leq \limsup _{k \rightarrow \infty} \sup _{b \geq k}\left(E \exp \left(\left(m_{k} / b\right) \sqrt{k}-a m_{k} / 2\right)+E \exp \left(m_{k} /(2 a)\right) I\{|X|>\sqrt{k}\}\right) \\
& \leq \limsup _{k \rightarrow \infty}\left(E \exp \left(\left(m_{k} / \sqrt{k}\right)-a m_{k} / 2\right)+\exp \left(m_{k} / a\right) P\{|X|>\sqrt{k}\}\right) \\
& =0 \text {, }
\end{aligned}
$$

as desired.

Put

$$
r_{\delta}(b, x)=\inf _{t \geq 0} \exp \left((t \delta)^{2} / 2\right) E \exp \left(t\left(b X-x\left(X^{2}+b^{2}\right) / 2\right)\right) .
$$

We next prove that $r_{\delta}(b, x)$ is a continuous function of $(b, x)$ over $[0, k] \times[a, 1]$ for every fixed $0<\delta<1$ and for any $k \geq 1$. Let $Y$ be a standard normal random variable. Put

$$
\xi_{b, x}:=\xi_{b, x, \delta}=b X+\delta Y-x\left(X^{2}+b^{2}\right) / 2 .
$$

Take $A_{0} \geq 1$ such that $P\left(|X| \leq A_{0}\right) \geq 1 / 2$. Then, we have

$$
r_{\delta}(b, x)=\inf _{t \geq 0} E \exp \left(t \xi_{b, x}\right) .
$$

Similarly to (2.12), there is $0<t_{b, x}<\infty$ such that

$$
r_{\delta}(b, x)=E \exp \left(t_{b, x} \xi_{b, x}\right) \leq 1 .
$$

Along the same lines of the proof of (2.13),

$$
t_{b, x} \leq\left(2 b A_{0}+x A_{0}^{2}+x b^{2}+2\right) / \delta^{2} \leq 6 k^{2} A_{0}^{2} / \delta^{2}
$$

for $0 \leq b \leq k$ and $a \leq x \leq 1$. Put

$$
T:=T(k, \delta)=6 k^{2} A_{0}^{2} / \delta^{2} .
$$

By (A.3), for any $0 \leq b, d \leq k, a \leq x, y \leq 1,0<\delta<1$ and $A \geq 1$,

$$
\begin{aligned}
r_{\delta}(b, x) \leq & E \exp \left(t_{d, y} \xi_{b, x}\right) \\
= & \exp \left(\left(\delta t_{d, y}\right)^{2} / 2\right) E \exp \left(t_{d, y}\left(b X-x\left(X^{2}+b^{2}\right) / 2\right)\right) I\{|X| \leq A\} \\
& +\exp \left(\left(\delta t_{d, y}\right)^{2} / 2\right) E \exp \left(t_{d, y}\left(b X-x\left(X^{2}+b^{2}\right) / 2\right)\right) I\{|X|>A\}
\end{aligned}
$$




$$
\begin{aligned}
\leq & \exp \left((|b-d|+|x-y|) T\left(A^{2}+k^{2}\right)\right) \exp \left(\left(\delta t_{d, y}\right)^{2} / 2\right) \\
& \times E \exp \left(t_{d, y}\left(d X-y\left(X^{2}+d^{2}\right) / 2\right)\right) \\
& +\exp \left(T^{2}+T k^{2} / a\right) P(|X|>A) \\
= & \exp \left((|b-d|+|x-y|) T\left(A^{2}+k^{2}\right)\right) r_{\delta}(d, y) \\
& +\exp \left(T^{2}+T k^{2} / a\right) P(|X|>A) \\
\leq & r_{\delta}(d, y)+\exp \left((|b-d|+|x-y|) T\left(A^{2}+k^{2}\right)\right)-1 \\
& +\exp \left(T^{2}+T k^{2} / a\right) P(|X|>A) .
\end{aligned}
$$

Therefore

$$
\begin{array}{r}
\left|r_{\delta}(b, x)-r_{\delta}(d, y)\right| \leq \exp \left((|b-d|+|x-y|) T\left(A^{2}+k^{2}\right)\right) \\
-1+\exp \left(T^{2}+T k^{2} / a\right) P(|X|>A),
\end{array}
$$

from which the continuity of $r_{\delta}(b, x)$ follows, by the fact that $\lim _{A \rightarrow \infty} P(|X|>$ $A)=0$. Hence, in terms of Dini's theorem [cf. Royden (1968), page 162] and Lemma 4 of Chernoff (1952),

$$
\begin{aligned}
& \lim _{\delta \downarrow 0} r_{\delta}(b, x)=\inf _{t \geq 0} E \exp \left(t\left(b X-x\left(X^{2}+b^{2}\right) / 2\right)\right) \\
& \text { uniformly in }(b, x) \in[0, k] \times[a, 1] .
\end{aligned}
$$

Consequently,

$$
\begin{gathered}
\lim _{\delta \downarrow 0} \sup _{0 \leq b \leq k} \inf _{t \geq 0} \exp \left((t \delta)^{2} / 2\right) E \exp \left(t\left(b X-x\left(X^{2}+b^{2}\right) / 2\right)\right) \\
=\sup _{0 \leq b \leq k} \inf _{t \geq 0} E \exp \left(t\left(b X-x\left(X^{2}+b^{2}\right) / 2\right)\right)
\end{gathered}
$$

uniformly in $x \in[a, 1]$ for any $k \geq 1$. Equation (2.16) now follows from (A.1) and (A.4).

Proof of Lemma 8.1. Clearly,

$$
f(x) \leq f_{\delta}(x) \leq 1 \text { for every } x \geq 0 .
$$

Consider $E X^{2}<\infty$ first. Put $b_{0}=\sqrt{E X^{2}}$. It is easy to see that

$$
\left.\frac{\mathrm{d}}{\mathrm{d} t} E \exp \left(t\left(b_{0} X-x_{0}\left(X^{2}+b_{0}^{2}\right) / 2\right)\right)\right|_{t=0}=0 .
$$

Recalling that $E \exp \left(t\left(b_{0} X-x_{0}\left(X^{2}+b_{0}^{2}\right) / 2\right)\right)$ is a convex function of $t$, we have

$$
\begin{aligned}
f\left(x_{0}\right) & \geq \inf _{t \geq 0} E \exp \left(t\left(b_{0} X-x_{0}\left(X^{2}+b_{0}^{2}\right) / 2\right)\right) \\
& =\left.E \exp \left(t\left(b_{0} X-x_{0}\left(X^{2}+E X^{2}\right) / 2\right)\right)\right|_{t=0}=1 .
\end{aligned}
$$

This proves (8.3). 
If $E X^{2}=\infty$, then $x_{0}=0$ and

$$
f(0)=\operatorname{supinf}_{b \geq 0} E \exp (t b X)=1
$$

as desired.

Observe that

$$
f(1)=\sup _{b \geq 0} \inf _{t \geq 0} E \exp \left(-t(X-b)^{2} / 2\right)=\sup _{b \geq 0} P(X=b) .
$$

So, (8.4) holds.

To prove the continuity and monotonicity of $f_{\delta}(x)$, let $Y$ be a standard normal random variable and independent of $X$. Then

$$
f_{\delta}(x)=\operatorname{supinf}_{b \geq 0} E \exp \left(t\left(\delta Y+b X-x\left(X^{2}+b^{2}\right) / 2\right)\right) .
$$

For every $x>x_{0}$, from the proof of Lemma 2.1,

$$
\liminf _{b \rightarrow \infty} E \exp \left(t\left(\delta Y+b X-x\left(X^{2}+b^{2}\right) / 2\right)\right)=0
$$

and $\inf _{t \geq 0} E \exp \left(t\left(\delta Y+b X-x\left(X^{2}+b^{2}\right) / 2\right)\right)$ is a continuous function of $b$. Therefore, for $x_{0}<x<y$, there exists $b_{y} \geq 0$ such that

$$
f_{\delta}(y)=\inf _{t \geq 0} E \exp \left(t\left(\delta Y+b_{y} X-y\left(X^{2}+b_{y}^{2}\right) / 2\right)\right) .
$$

Since $\delta Y+b_{y} X-x\left(X^{2}+b_{y}^{2}\right) / 2$ is a continuous random variable, and $E(\delta Y+$ $\left.b_{y} X-x\left(X^{2}+b_{y}^{2}\right) / 2\right)<0$, there exists $t_{x, y}>0$ such that

$$
\begin{aligned}
& \inf _{t \geq 0} E \exp \left(t\left(\delta Y+b_{y} X-x\left(X^{2}+b_{y}^{2}\right) / 2\right)\right) \\
&=E \exp \left(t_{x, y}\left(\delta Y+b_{y} X-x\left(X^{2}+b_{y}^{2}\right) / 2\right)\right) .
\end{aligned}
$$

A combination of (A.5) and (A.6) yields

$$
\begin{aligned}
f_{\delta}(y) & \leq E \exp \left(t_{x, y}\left(\delta Y+b_{y} X-y\left(X^{2}+b_{y}^{2}\right) / 2\right)\right) \\
& <E \exp \left(t_{x, y}\left(\delta Y+b_{y} X-x\left(X^{2}+b_{y}^{2}\right) / 2\right)\right) \\
& =\inf _{t \geq 0} E \exp \left(t\left(\delta Y+b_{y} X-x\left(X^{2}+b_{y}^{2}\right) / 2\right)\right) \\
& \leq \operatorname{supinf}_{b \geq 0} E \exp \left(t\left(\delta Y+b X-x\left(X^{2}+b^{2}\right) / 2\right)\right) \\
& =f_{\delta}(x) .
\end{aligned}
$$

That is, $f_{\delta}(x)$ is strictly decreasing for $x>x_{0}$.

We finally prove the continuity of $f_{\delta}(x)$. Given $x>x_{0}$ From the proof of (A.1) it follows that

$$
\lim _{k \rightarrow \infty} \operatorname{supinf}_{b \geq k} \exp \left(t^{2} / 2\right) E \exp \left(t\left(b X-y\left(X^{2}+b^{2}\right) / 2\right)\right)=0,
$$


uniformly in $y \geq\left(x+x_{0}\right) / 2$. Also, there are $0 \leq b_{x} \leq k$ and $t_{x, y}>0$ such that

$$
\begin{aligned}
\sup _{0 \leq b \leq k} & \inf _{t \geq 0} \exp \left((t \delta)^{2} / 2\right) E \exp \left(t\left(b X-x\left(X^{2}+b^{2}\right) / 2\right)\right) \\
& =\inf _{t \geq 0} \exp \left((t \delta)^{2} / 2\right) E \exp \left(t\left(b_{x} X-x\left(X^{2}+b_{x}^{2}\right) / 2\right)\right) \\
& =\exp \left(\left(t_{x, y} \delta\right)^{2} / 2\right) E \exp \left(t_{x, y}\left(b_{x} X-y\left(X^{2}+b_{x}^{2}\right) / 2\right)\right) .
\end{aligned}
$$

It follows from (A.3) that

$$
0 \leq t_{x, y} \leq T:=k^{2} A_{0}^{2}(3+x) / \delta^{2} \text { for all }|y-x| \leq 1
$$

and hence for $x \leq y \leq x+1$

$$
\begin{aligned}
f_{\delta}(y) \leq & f_{\delta}(x) \\
\leq & \exp \left(\left(t_{x, y} \delta\right)^{2} / 2\right) E \exp \left(t_{x, y}\left(b_{x} X-x\left(X^{2}+b_{x}^{2}\right) / 2\right)\right) \\
= & \exp \left(\left(t_{x, y} \delta\right)^{2} / 2\right) E \exp \left(t_{x, y}\left(b_{x} X-x\left(X^{2}+b_{x}^{2}\right) / 2\right)\right) I\{|X| \leq A\} \\
& +\exp \left(\left(t_{x, y} \delta\right)^{2} / 2\right) E \exp \left(t_{x, y}\left(b_{x} X-x\left(X^{2}+b_{x}^{2}\right) / 2\right)\right) I\{|X|>A\} \\
\leq & \exp \left(t_{x, y}(y-x)\left(A^{2}+k^{2}\right)\right) \exp \left(\left(t_{x, y} \delta\right)^{2} / 2\right) \\
& \times E \exp \left(t_{x, y}\left(b_{x} X-x\left(X^{2}+b_{x}^{2}\right) / 2\right)\right) \\
& +\exp \left((T \delta)^{2}\right) \exp \left(T k^{2} / x\right) P(|X|>A) \\
\leq & \exp \left(T(y-x)\left(A^{2}+k^{2}\right)\right) f_{\delta}(y)+\exp \left((T \delta)^{2}\right) E \exp \left(T k^{2} / x\right) P(|X|>A) \\
\leq & f_{\delta}(y)+\exp \left(T(y-x)\left(A^{2}+k^{2}\right)\right)-1 \\
& +\exp \left((T \delta)^{2}\right) \exp \left(T k^{2} / x\right) P(|X|>A) .
\end{aligned}
$$

This proves $\lim _{y \downarrow x} f_{\delta}(y)=f_{\delta}(x)$. Similarly, one has $\lim _{y \uparrow x} f_{\delta}(y)=f_{\delta}(x)$. This proves the continuity of $f_{\delta}(x)$. Also, one can prove the right continuity of $f_{\delta}(x)$ at $x=x_{0}$. The continuity of $f(x)$ is a direct consequence of Lemma 2.1 and the continuity of $f_{\delta}(x)$.

Acknowledgments. The author would like to thank Professor Xuming He for his many helpful suggestions on the paper. Many thanks are also due to an anonymous referee for very useful comments to improve the presentation of the paper.

\section{REFERENCES}

BAHADUR, R. R. (1971). Some limit theorems in statistics. Regional Conference Series in Applied Mathematics 4. SIAM, Philadel phia.

Bingham, N. H., Goldie, C. M. and Teugels, J. L. (1987). Regular Variation. Cambridge Univ. Press.

Chernoff, H. (1952). A measure of asymptotic efficiency for tests of a hypothesis based on the sum of observations. Ann. Math. Statist. 23 493-507.

Chung, K. L. (1974). A Course in Probability Theory. Academic Press, New York. 
CRAMÉR, H. (1938). Sur un nouveaux théoréme limite de la théorie des probabilités. Actualités Sci. Indust. 736 5-23. Hermann, Paris.

CsÖRGÖ, M. and HoRváth, L. (1988). Asymptotic representations of self-normalized sums. Probab. Math. Statist. 9 15-24.

CsÖRGŐ, M. and RÉVÉSZ, P. (1981). Strong Approximations in Probability and Statistics. Academic Press, New York.

CsÖRGŐ, M. and SHAO, Q. M. (1994). A self-normalized Erdős-Rényi type strong law of large numbers. Stochastic Process. Appl. 50 187-196.

CsÖRGŐ, M. and STEINEBACH, J. (1981). Improved Erdős-Rényi and strong approximation laws for increments of partial sums. Ann. Probab. 9 988-996.

CsöRGö, S. (1979). Erdős-Rényi laws. Ann. Statist. 7 772-787.

DARLING, D. A. (1952). The influence of the maximum term in the addition of independent random variables. Trans. Amer. Math. Soc. 73 95-107.

De Acosta, A. (1988). Large deviations for vector-valued functionals of a Markov chain: lower bounds. Ann. Probab. 16 925-960.

Deheuvels, P., Devroye, L. and LyNCH, J. (1986). Exact convergence rate in the limit theorems of Erdős-Rényi and Shepp. Ann. Probab. 14 209-223.

Dembo, A. and Zeitouni, O. (1992). Large Deviations Techniques and Applications. J ones and Bartlett, Boston.

Donsker, M. D. and VARADHAN, S. R. S. (1987). Large deviations for noninteracting particle systems. J . Statist. Physics 46 1195-1232.

EFron, B. (1969). Student's $t$-test under symmetry conditions. J . Amer. Statist. Assoc. 64 12781302.

ERDŐs, P. and RÉNYI, A. (1970). On a new law of large numbers. J . Analyse Math. 23 103-111.

FELLER, W. (1966). An Introduction to Probability Theory and Its Applications. Wiley, New York.

GRIFFIN, P. and KuELBS, J. (1989). Self-normalized laws of the iterated logarithm. Ann. Probab. 17 1571-1601.

Hahn, M. G., Kuelbs, J. and Weiner, D. C. (1990). The asymptotic joint distribution of selfnormalized censored sums and sums of squares. Ann. Probab. 18 1284-1341.

Hotelling, H. (1961). The behavior of some standard statistical tests under nonstandard conditions. Proc. Fourth Berkeley Symp. Math. Statist. Probab. 1 319-360. Univ. California Press, Berkeley.

KARAMATA, J. (1933). Sur un mode de croissance régulière, théorèmes fondamentaux. Bull. Soc. Math. France 61 55-62.

Logan, B. F., Mallows, C. L., Rice, S. O. and Shepp, L. A. (1973). Limit distributions of selfnormalized sums. Ann. Probab. 1 788-809.

Petrov, V. V. (1965). On the probabilities of large deviations for sums of independent random variables. Theory Probab. Appl. 10 287-298.

Petrov, V. V. (1975). Sums of Independent Random Variables. Springer, New York.

Petrov, V. V. and S̆Irokova, I. V. (1973). The exponential rate of convergence in the law of large numbers. Vestnik Leningrad. Univ. No. 7 Mat. Mekh. Astronom. 2 155-157.

Royden, H. L. (1968). Real Analysisi, 2nd ed. Macmillan, New York.

Shepp, L. A. (1966). A limit theorem concerning moving averages. Ann. Math. Statist. 35 424-428.

SteINEBACH, J. (1980). Large deviation probabilities and some related topics. Carleton Math. Lecture Notes 28. Carleton Univ.

Strassen, V. (1966). A converse to the law of the iterated logarithm. Z. Wahrsch. Verw. Gebiete $4265-268$.

StRoock, D. W. (1984). An Introduction to the Theory of Large Deviations. Springer, Berlin.

DePARTMENT OF MATHEMATiCS

UNIVERSITY OF OREGON

EugENE, OREGON 97403

E-MAIL: shao@math.uoregon.edu 